\title{
Project Finance y Asociaciones Público-Privada para la provisión de servicios de infraestructura en Colombia
}

\author{
Project Finance and Public-Private Partnerships for the provision of infrastructure services in \\ Colombia
}

Fecha de entrega: 14 de septiembre 2014

Fecha de aceptación: 27 de noviembre 2014

\section{Juan D. González ${ }^{1}$, Miguel D. Rojas², Carlos A. Arboleda ${ }^{3}$ y Sergio Botero $^{4}$}

1 Dirección de Empresas, Institución Universitaria Esumer, Universidad Nacional de Colombia, Calle 76 Nº. 80-126, Bloque 1 Piso 2, Medellín, Colombia,jdgonzalez@esumer.edu.co,jdgonza3@unal.edu.co

2 Centro de Investigación y Consultoría Organizacional CINCO, Facultad de Minas, Bloque M8B, oficina 106, Universidad Nacional de Colombia, Carrera 80 No.65-223, Medellín, Colombia, mdrojas@unal.edu.co

3 Gerente Infraestructura, Pedro Gómez y Cia, Calle 70 No 7 - 53, Bogotá, Colombia, aarboleda@pedrogomez.com.co

${ }^{4}$ Modelamiento y Análisis, Energía, Ambiente y Economía, Facultad de Minas, Bloque M8B, oficina 106, Universidad Nacional de Colombia, Carrera 80 No.65-223, Medellín, Colombia, sbotero@unal.edu.co

El Project Finance PF y las Asociaciones Público-Privadas APPs son utilizados como instrumentos de financiación de proyectos y medio para vincular el sector privado en el desarrollo de infraestructura pública. En el presente trabajo se realiza una revisión de la literatura y los asuntos actuales del PF y las APPs para la provisión de servicios de infraestructura en Colombia, enunciando las principales relaciones y características en el desarrollo de proyectos. Se revisaron fuentes especializadas categorizadas en el Journal Citation Reports JCR, así como publicaciones de organismos multilaterales y nacionales relacionados con desarrollo de proyectos de infraestructura. Se realiza una caracterización de PF describiendo las etapas, riesgos, mecanismos de control y seguimiento y se explica la diferencia con la forma tradicional de financiar proyectos, posteriormente se detallan las principales ventajas y desventajas de las APP especificamente en el marco de la Ley 1508 de 2012. Finalmente, se enuncian las lineas actuales de investigación y, además, se concluye con el planteamiento de futuras lineas para el contexto colombiano que conduzcan a cerrar la brecha entre la teoría y la práctica.

Palabras clave: Asociación Público-Privadas, Colombia, financiación de proyectos, infraestructura, investigación
Project Finance PF and Public-Private Partnerships PPP are used as project financing tools and a means to link the private sector with the public infrastructure development. In this work a literature review and current matters of PF and PPP for the provision of infrastructure services in Colombia are pursued, outlining the main relationships and characteristics in the developing of projects. Specialised sources, categorised in the Journal Citation Reports JCR, were reviewed, as well as publications from multilateral and national organisations related with the development of infrastructure projects. A characterisation of PF is carried out, describing the stages, risks, control and tracking mechanisms. The difference with the traditional form of project financing is explained. Then, advantages and disadvantages of APPS within the framework of the Law 1508 from 2012 are in detail described. Finally, in view of the gap between theory and practice, the current and future research lines within the Colombian context are pointed out.

Keywords: Public-Private Partnerships, Colombia, project finance, infrastructure, research 


\section{Introducción}

De acuerdo con Guasch (2004) y Saussier (2013), la participación del sector privado en el desarrollo de infraestructura social y productiva es motivada por la necesidad de mejorar el funcionamiento y la cobertura de bienes y servicios, lo cual debido a la escasez de fondos públicos y restricciones en la capacidad de endeudamiento, varios países optan por transferir la prestación de infraestructura al sector privado (ver también AlonsoConde et al., 2007; Jin y Zhang, 2011; Thomson et al., 2005; Zhang y Durango-Cohen, 2012). Esto beneficia el mejoramiento en los niveles y la calidad, lo cual genera un enorme efecto sobre el crecimiento económico y la disminución de la pobreza; así para Vasallo (2012) estas condiciones justifican la elaboración de análisis y enfoques que sean capaces de contribuir al desarrollo de una nueva visión desde las finanzas públicas, el sector financiero y las empresas constructoras, por medio de nuevas estrategias y visión de diferente modelos de financiación.

Tabla 1: Tipo de infraestructura, sector y tipo de proyecto, basado en Grimsey y Lewis (2002) y Perroti y Sánchez (2011)

\begin{tabular}{|l|l|l|}
\hline Infraestructura & \multicolumn{1}{|c|}{ Sector } & \multicolumn{1}{c|}{ Tipo de proyecto } \\
\hline \multirow{5}{*}{ Social } & $\begin{array}{l}\text { Agua y } \\
\text { saneamiento }\end{array}$ & $\begin{array}{l}\text { Acueducto, alcantarillado, } \\
\text { disposición de residuos sólidos, } \\
\text { tratamiento de aguas residuales, } \\
\text { presas y embalses }\end{array}$ \\
\cline { 2 - 3 } & $\begin{array}{l}\text { Educación } \\
\text { Salud }\end{array}$ & $\begin{array}{l}\text { Escuelas y universidades } \\
\text { Hospitales y centros de salud }\end{array}$ \\
\cline { 2 - 3 } & Defensa & $\begin{array}{l}\text { Cárceles y centros de detención } \\
\text { preventiva }\end{array}$ \\
\cline { 2 - 4 } & $\begin{array}{l}\text { Edificación } \\
\text { pública }\end{array}$ & $\begin{array}{l}\text { Oficinas del sector público y del } \\
\text { poder judicial }\end{array}$ \\
\cline { 2 - 4 } & $\begin{array}{l}\text { Deportivo y } \\
\text { cultural }\end{array}$ & $\begin{array}{l}\text { Museos, parques, centros de } \\
\text { recreación y culturales }\end{array}$ \\
\hline \multirow{5}{*}{ Productiva } & Energía & $\begin{array}{l}\text { Plantas de generación y } \\
\text { suministro, redes de distribución }\end{array}$ \\
\cline { 2 - 4 } & $\begin{array}{l}\text { Transporte } \\
\text { caciones }\end{array}$ & $\begin{array}{l}\text { Carreteras de peaje, puertos, } \\
\text { aeropuertos, sistemas ferroviarios, } \\
\text { puentes y túneles }\end{array}$ \\
\cline { 2 - 4 } & Telefonía, redes y satélites \\
\hline
\end{tabular}

En esta misma línea, Moszoro y Gasiorowski (2008) mostraron con un análisis basado en modelos que "las asociaciones público-privadas pueden prestar los servicios públicos más baratos que una entidad pública o privada única", considerando todo el ciclo de vida del proyecto y agregan que "las consideraciones de eficiencia sugieren que la propiedad de la sociedad vehículo que presta el servicio público no tiene por qué ser exclusivamente pública o privada. Una inversión óptima en la infraestructura pública requiere la propiedad mixta"(p.13). De acuerdo con Grimsey y Lewis (2002), la infraestructura es fácil de reconocer pero difícil de definir, así la infraestructura se define como la inversión que proporciona los servicios básicos a la industria y los hogares, así como los insumos claves para la economía; aunque lo básico, puede variar de país a país y de un tiempo a otro.

Adicionalmente, el reciente crecimiento de las Asociaciones Público- Privadas APPs está estrechamente relacionado con la técnica de financiación conocida como Project Finance $P F$, dadas las características que la hacen adecuada para este tipo de proyectos (Yescombe, 2007). Por su parte Ghersi y Sabal (2006) afirman que el $P F$ es utilizado ampliamente en mercados emergentes por las empresas multinacionales que desean limitar su exposición a los diferentes riesgos en el país, además, las instituciones multilaterales pueden desempeñar un papel clave en la financiación de proyectos, los préstamos son vistos como menos riesgosos y terminan siendo menos costosos que los préstamos bancarios; así, la participación como banqueros se interpreta como una señal positiva por los bancos comerciales quienes estarán dispuestos en participar en la financiación por medio de la sindicación de créditos. Sin embargo, en el caso de un proyecto estatal, se debe considerar que la deuda soberana es menos costosa que la deuda que enfrentará un Vehículo de Propósito Especial.

Se considera que una de las ventajas de las APPs es el empaquetamiento de actividades, lo cual permite tener beneficios en términos de costos, transferencia de riesgos y mejoras en la planeación de los proyectos, entre otros (Engel et al., 2010; Zhang y Chen, 2013). Para Guasch (2014), el efecto empaquetamiento soluciona, por ejemplo, el problema de rehabilitación y mantenimiento debido a las economías de escala que se puedan presentar, impactando la reducción de costos y tiempos de construcción, la calidad de la infraestructura y los niveles de servicio; así como incrementos en la productividad, lo cual se evidencia 
en más de 20 años de investigación. Una crítica respecto al empaquetamiento se fundamenta en la concentración de actividades, lo cual podría traer consecuencias negativas en caso de liquidación o deficiente operación, teniendo resultados inesperados desde la óptica social y financiera de la infraestructura.

Por otra parte, los proyectos desarrollados bajos los esquemas planteados en este artículo se caracterizan por tener asociados una gran cantidad de contratos; por lo cual, las renegociaciones se consideran como una de las principales desventajas. Considerando que el estudio de grandes proyectos de infraestructura tiene la capacidad de generar una visión académica que contribuya a mejorar la práctica actual (Esty, 2004), la identificación exacta del efecto de la eficiencia del $P F$ y las APPs en el sector de infraestructura, ofrece una vía pertinente para futuras investigaciones (Kleimeier y Versteeg, 2010). En este sentido, es recomendable que se coordinen las estrategias de desarrollo de infraestructura junto con el mercado de capitales, lo que debería complementarse mediante el fortalecimiento de las capacidades de planificación y financiamiento de proyectos (Rozas et al., 2012).

Las evidencias presentadas por los investigadores Kayser (2013), Gatti (2013), Tang et al. (2010), Kleimeier (1995), Kleimeier et al. (2010), Rosillo y Perdomo (2012), Esty
(2004), Hinojosa (2009, 2010) y Cruz et al. (2014) argumentan la necesidad de investigar sobre la relación entre $P F$ y APPs por el potencial en establecer nuevos esquemas de colaboración entre sectores público-privado, así como la creación de nuevas teorías financieras. Con la apertura de los mercados mundiales y la necesidad de generar nuevas y mejores formas de hacer negocios, implica que se debe estudiar cómo y de qué manera se estructuran modelos eficientes que busquen maximizar la rentabilidad con una transferencia adecuada de riesgos entre los participantes; implicando medir el impacto de dichas estructuras, así como la conveniencia de aplicación en los diferentes sectores de la economía.

El artículo está organizado de la siguiente manera: después de la presente introducción, se expone la metodología, donde se describen el proceso metodológico para la búsqueda y sistematización de la información. Luego, se presenta el marco teórico donde se incluye la revisión de la literatura y las principales características de la Ley 1508 de 2012 la cual reglamenta las APPs en Colombia. Finalmente se exponen los resultados de la investigación presentando el estado de la cuestión; las conclusiones incluyen las futuras líneas de investigación propuestas derivadas del análisis.

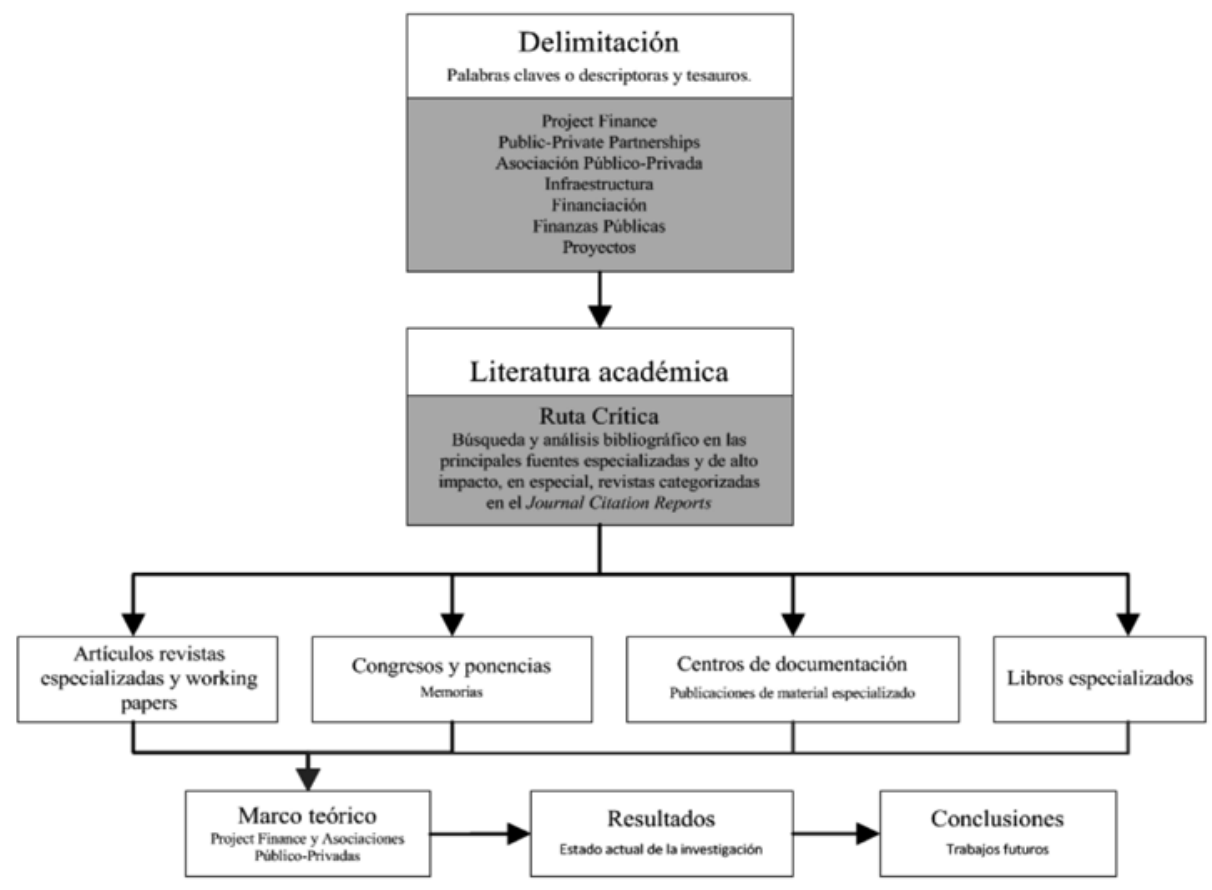

Figura 1: Esquema metodológico para desarrollar la investigación (basado en Esquivel, 2013) 
Metodología

En este artículo se ha empleado la metodología sugerida por Esquivel (2013), buscando establecer los lineamientos y relaciones generales del $P F$ y las APPs. Se planteó como ruta crítica la búsqueda y análisis bibliográfico en las principales fuentes especializadas y de alto impacto; en especial, revistas categorizadas en el Journal Citation Reports JCR, así como en repositorios de tesis de posgrados, libros, informes, investigaciones y documentos; que permitieron desarrollar de manera sistemática la presente investigación; incluyendo: marco teórico, avances, tendencias y elementos de relevancia teórica y práctica que permiten determinar el estado de la cuestión y sugerir futuras líneas de investigación (Figura 1).

\section{Marco teórico}

En esta sección se presenta la diferencia entre el Project Finance $P F$ y la financiación tradicional; se presentan además, los principales riesgos asociados en el desarrollo de proyectos de infraestructura, así como mecanismos de control y seguimiento utilizados por los promotores (sponsor); adicionalmente se resalta la importancia de los créditos sindicados y la participación de los bancos de desarrollo en la financiación. Posteriormente, se presenta una revisión de las APPs y se enuncian los principales esquemas de participación del sector privado para la provisión de infraestructura en Colombia. Adicionalmente, se presentan las ventajas y desventajas.

\section{Project Finance: técnica de financiación y estructuración de proyectos de infraestructura}

La Tabla 2 presenta los principales aportes a la definición de PF. Respecto al endeudamiento por fuera de balance, Mascareñas (1999) afirma que las normas de consolidación de estados financieros son claras al respecto: en el balance individual del promotor no aparece la deuda asociada al $\mathrm{PF}$; sin embargo, por el método contable que corresponda tendrá que aparecer en el balance consolidado del grupo que forma parte la entidad creada para el desarrollo del proyecto. Cuando el promotor avala o garantiza la obligación financiera del $P F$, ésta deberá ser contabilizada en los estados financieros del promotor, porque en el caso que el proyecto no cumpla con el pago de la obligación financiera, el promotor deberá responder por lo establecido
Tabla 2: Principales aportes a la definición de Project Finance

\begin{tabular}{|c|c|}
\hline Referencia & Definición \\
\hline Gatti (2013) & $\begin{array}{l}\text { Financiación estructurada de una entidad } \\
\text { económicamente independiente, creada por el } \\
\text { promotor, usando recursos propios y deuda, en } \\
\text { la cual los prestamistas consideran los flujos de } \\
\text { caja como fuente primaria para el reembolso } \\
\text { del crédito, mientras que los activos del } \\
\text { proyecto representan la garantía (collateral). }\end{array}$ \\
\hline Esty (2003) & $\begin{array}{l}\text { Financiación de capital económicamente } \\
\text { independiente y de vida limitada, diseñado } \\
\text { en función de la caja estimada y de las } \\
\text { características particulares del proyecto, } \\
\text { proporcionando retorno al capital invertido, } \\
\text { teniendo como garantía los activos de la } \\
\text { unidad económica y los recursos económicos } \\
\text { generados por el proyecto. }\end{array}$ \\
\hline $\begin{array}{l}\text { Durán } \\
\text { (2006) }\end{array}$ & $\begin{array}{l}\text { Técnica de financiación para la ejecución } \\
\text { de proyectos de infraestructura, utilizando } \\
\text { un vehículo de inversión legalmente } \\
\text { independiente del promotor (Special Purpose } \\
\text { Vehicle } S P V \text { ), el cual se podría constituir por } \\
\text { medio de una sociedad comercial o un contrato } \\
\text { de fiducia mercantil, cuyo propósito principal } \\
\text { se fundamenta en que los financiadores del } \\
\text { proyecto obtengan el servicio a la deuda } \\
\text { con la garantía de los flujos de caja futuros } \\
\text { generados por el proyecto; de esa manera el } \\
\text { endeudamiento se realiza por fuera de balance. }\end{array}$ \\
\hline $\begin{array}{l}\text { Gómez } \\
\text { y Jurado } \\
\text { (2001) }\end{array}$ & $\begin{array}{l}\text { Financiación de un proyecto sin recursos } \\
\text { o con recursos limitados al promotor, cuya } \\
\text { amortización de capital y pago de intereses se } \\
\text { realiza con el flujo de caja. }\end{array}$ \\
\hline $\begin{array}{l}\text { Molina et } \\
\text { al. (2004) }\end{array}$ & $\begin{array}{l}\text { Argumentan que no hay una definición } \\
\text { aceptada sobre el } P F \text {, pero, a su juicio, es un } \\
\text { sistema de financiamiento que permite que } \\
\text { un determinado proyecto de inversión, dadas } \\
\text { sus posibilidades de generar flujos de caja } \\
\text { razonablemente predecibles y sostenibles, } \\
\text { junto a la calidad de sus propios activos, } \\
\text { sea financiado individualmente, utilizando } \\
\text { específicamente los fondos obtenidos por el } \\
\text { proyecto, sin recurso pleno del promotor y } \\
\text { empleando el mayor apalancamiento posible. }\end{array}$ \\
\hline $\begin{array}{l}\text { Departa- } \\
\text { mento } \\
\text { Nacional de } \\
\text { Planeación } \\
\text { de } \\
\text { Colombia } \\
(2011 \mathrm{a}) \\
\end{array}$ & $\begin{array}{l}\text { Se fundamenta en Molina et al. (2004) y agrega } \\
\text { que los bancos y proveedores de patrimonio } \\
\text { basarán la decisión de prestar dinero en el } \\
\text { flujo de caja generado por el proyecto, sin } \\
\text { poder exigir pago alguno de los accionistas } \\
\text { de la compañías encargadas de desarrollar el } \\
\text { proyecto; es decir, non recourse. }\end{array}$ \\
\hline
\end{tabular}


en el contrato de deuda. En la Figura 2 se muestra la estructura típica de un proyecto de infraestructura desarrollado con la técnica del $P F$, donde éste se encuentra fuera del balance del promotor y por tanto el deudor será el Vehículo de Inversión.

Por otra parte, el promotor no otorga garantías personales ni corporativas, dado que afectan el balance por las contingencias que eso implica (Durán, 2006). Además, bajo este esquema la falta de ingresos del proyecto y por ende el incumplimiento de los compromisos financieros no habilita a las entidades financieras a solicitar recursos económicos al promotor (González, 2006). Tampoco tienen una relación contractual para reembolsar el aporte de capital realizado por el promotor al vehículo de

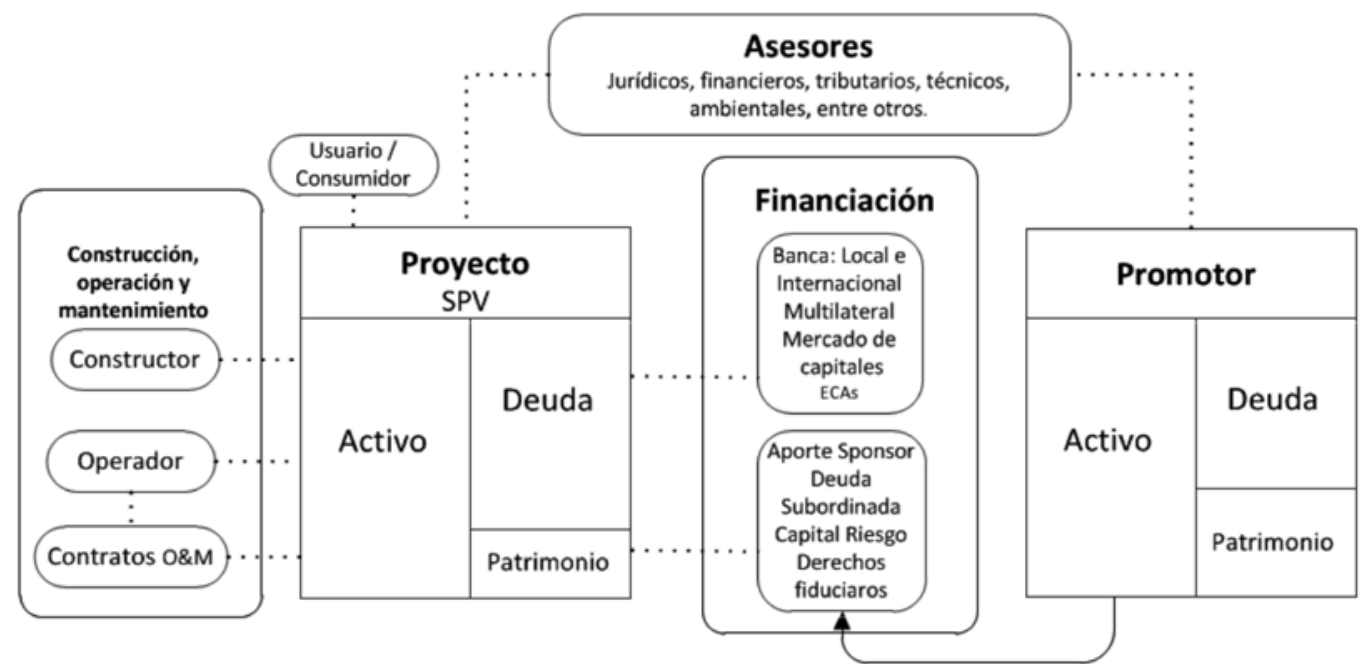

Figura 2: Relaciones contractuales y estructura típica de un PF, basado en Esty y Christov (2002), Grajales (2012), Gatti (2013) y Bracey y Moldovan (2006)

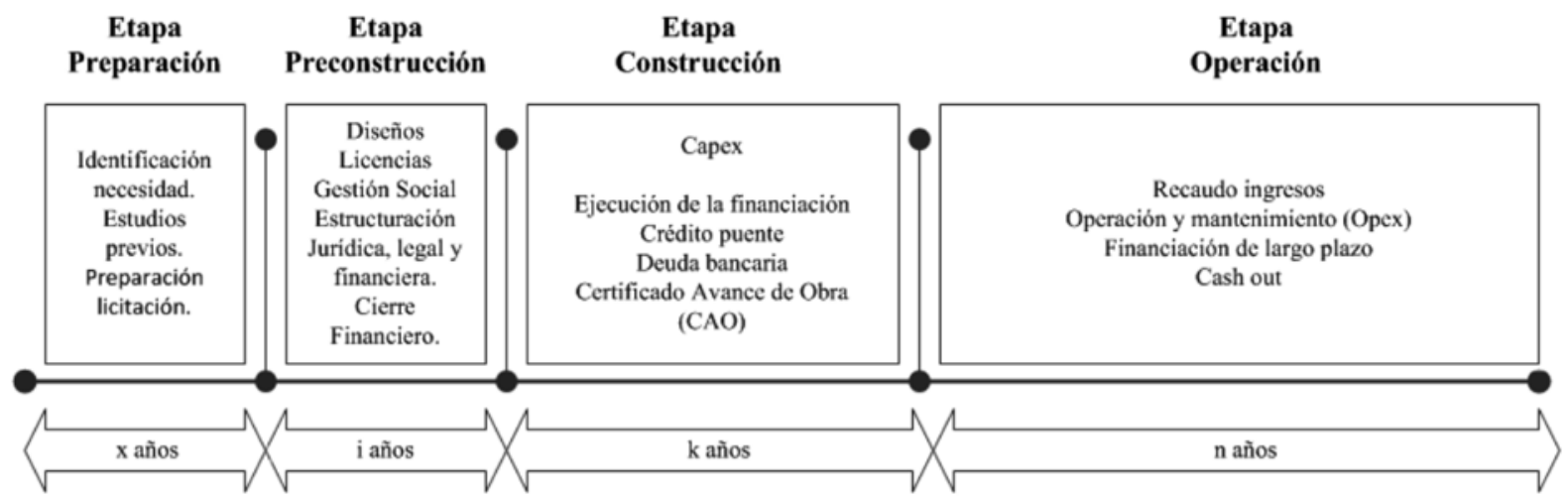

Figura 3: Etapas ciclo de vida PF, basado en Grajales (2012)
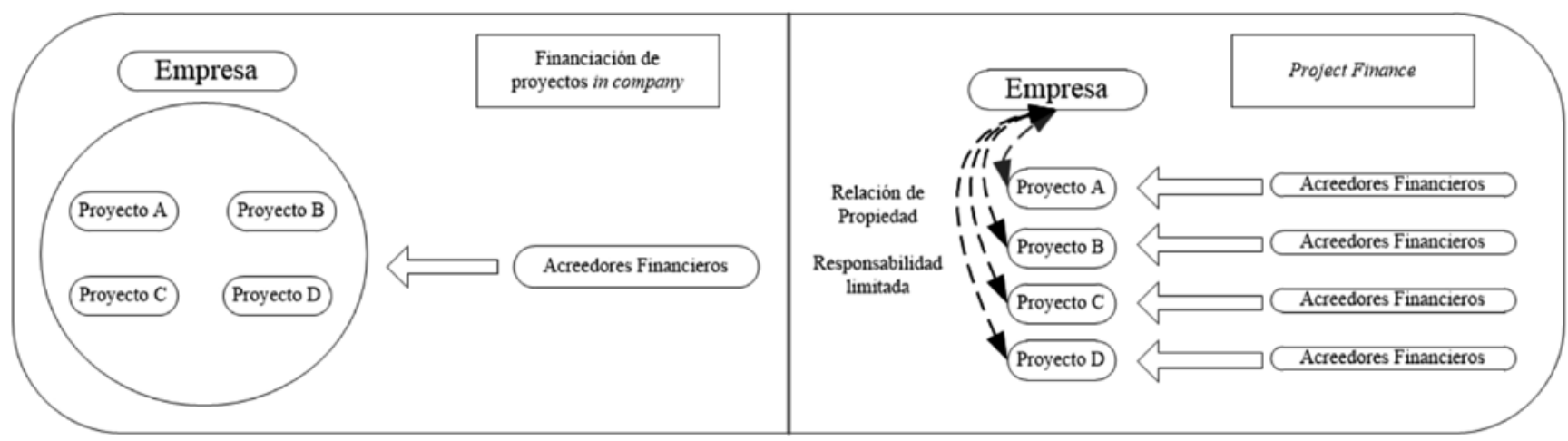

Figura 4: Financiación tradicional versus PF, basado en Suárez (1993) 
inversión; así, los prestamistas garantizan, en primera instancia, que el flujo de caja libre se utilice para cubrir las obligaciones financieras y no para remunerar al promotor, permitiéndolo solo cuando se produzcan excesos y el contrato de deuda lo permita (Molina y Del Carpio, 2004). Ellos confirman lo expuesto por otros autores como Esty (2003) y Gatti (2013), dado que se pretende que existan limitadas obligaciones de pago del promotor respecto a la deuda del proyecto (Gutiérrez y Bielenberg, 2009).

Es importante considerar que el $P F$ no es una alternativa de financiación cuando, en circunstancias normales, el proyecto no es financiable, bien por no generar tasas adecuadas de rendimiento o por cualquier otra circunstancia, dado que no es una alternativa para los casos en los cuales la financiación tradicional, no es viable (Mascareñas, 1999). Es decir, el $P F$ no convierte los proyectos inviables en viables. En el desarrollo de proyectos de infraestructura se identifican cuatro etapas, cada una tiene asociado eventos o actividades específicas las cuales se muestran en la Figura 3. Para el caso de Colombia la etapa de operación no puede superar los 30 años, incluyendo prorrogas (Congreso de Colombia, 2012a).

\section{Diferencia entre PF y financiación tradicional}

Respecto a la diferencia con los mecanismos tradicionales de financiamiento (Corporate Finance), los proyectos no suelen adquirir la condición jurídica de sociedades separadas; los pactos contractuales no son exhaustivos, los indicadores de endeudamiento sobre recursos propios no son altos y la mayoría de los préstamos ofrecen como garantía los activos del prestatario, en caso de impago (Molina y Del Carpio, 2004). Así, la ventaja del $P F$ respecto del financiamiento corporativo tradicional, consiste en que la deuda contraída por el proyecto, por medio del vehículo de inversión, no es considerada deuda corporativa, permitiendo al promotor implementar diversos proyectos sin llegar a su capacidad de endeudamiento (Gutiérrez et al., 2009), como se mostró en la Figura 2.

Por lo anterior, el tratamiento contable de la obligación financiera en esta modalidad de proyectos se considera fuera de balance (off-balance); así, las garantías que ofrece el promotor para fondear el proyecto son los activos y los flujos de caja futuros (Gatti, 2013; Durán, 2006). Reafirmando lo anterior, Suárez (1993) afirma que en el
$P F$, a diferencia de la forma tradicional de financiación de proyectos, la responsabilidad de la deuda recae parcial o totalmente en el proyecto, no en la organización o promotor que lo lleva a cabo. Por otra parte, es importante considerar los altos costos de transacción en proyectos desarrollados por medio de $P F$, los cuales pueden estar entre el $5 \%$ y el $10 \%$ del coste total del proyecto (Esty, 2004).

En la Figura 4 se muestra la diferencia entre la financiación tradicional y $P F$. Se evidencia en la parte izquierda que la empresa realiza sus proyectos in company, es decir, se encuentran dentro de su balance general el cual es impactado por los pasivos financieros de cada proyecto; en la derecha se muestra que la empresa desarrolla los proyectos en vehículos de inversión independientes, lo cual limita la responsabilidad del promotor, así como las obligaciones con los acreedores.

\section{Desarrollo de proyectos de APPs en Colombia por medio de PF}

El uso de $P F$ está adquiriendo protagonismo, en la actualidad se extiende a parques solares y eólicos, autopistas, hospitales, escuelas, universidades, cárceles, entre otros (Vega, 2008). Uno de los principios del $P F$ se fundamenta en que la estructura legal y financiera del proyecto logre tener menor riesgo en comparación con la financiación tradicional, igualmente se pretende asegurar el pago del servicio a la deuda con el flujo de caja libre generado por el proyecto, incluso bajo escenarios extremos. Por otra parte, se pueden establecer requisitos de prepago con una parte de los ingresos remanentes y otras protecciones como pactos o convenios (covenants), que permiten generar tranquilidad a los acreedores financieros (Hinojosa, 2010).

En Colombia, el Departamento Nacional de Planeación de Colombia DNP (2011b) argumenta que se debe fortalecer el desarrollo de proyectos de infraestructura ejecutados por el sector privado por medio de la implementación de esquemas de APPs, que aseguren una adecuada asignación de riesgos y permitan el financiamiento a través de la emisión de títulos de contenido crediticio y/o participativo en el mercado de capitales; aunque el sistema financiero colombiano tiene un desarrollo considerable en los últimos años, se requiere, en asociación con el sector privado, una política integral de largo plazo para el desarrollo y 
expansión, lo cual beneficiará la financiación de proyectos desarrollados por medio de la técnica del $P F$.

De esta manera y como instrumento para impulsar el desarrollo de proyectos de infraestructura, la Ley 1508 de 2012 en el artículo 6 indica que el sector privado deberá constituir un Patrimonio Autónomo, el cual servirá como Vehículo de Propósito Especial SPV, integrado por los pasivos y activos del proyecto (Congreso de Colombia, 2012a), de manera que el desarrollo de proyectos de infraestructura en Colombia se enmarca bajo el concepto de APPs y está estrechamente relacionada con la técnica de estructuración y financiamiento de proyectos conocida como $P F$.

Esta Ley fue decretada con el objetivo de impulsar la vinculación de capital privado para la provisión de infraestructura pública y establece en el artículo 25 que los proyectos se deben registrar en el Registro Único de Asociaciones Público-Privadas RUAPP, lo cual permitirá tener transparencia sobre los procesos de celebración y adjudicación de contratos de los proyectos, incluyendo sus estados de desarrollo. Desde la expedición realizada el 10 de enero de 2012 hasta el tercer trimestre del 2014 se han registrado 273 proyectos, de los cuales 83 han sido rechazados. El DNP ha identificado que los principales motivos de rechazo son (DNP, 2014): requerir más del 20\% de recursos públicos, la infraestructura no se encuentra dentro de los planes de desarrollo, no existe una adecuada distribución de los riesgos entre el sector público y privado, no contempla la transferencia de la infraestructura al sector público, no incorpora la premisa que la remuneración se realiza cuando la infraestructura esté disponible y no cumple con los estándares de calidad o servicio.

Respecto a los proyectos de transporte, representan el $40.8 \%$ de los registrados en el RUAPP (DNP, 2014), ésto se debe en gran medida a la expedición de Ley 1682 de 2013, la cual reglamenta los proyectos de infraestructura de transporte buscando aumentar la cobertura y mejorar los niveles de competitividad por medio de las APPs (Fedesarrollo, 2012). De esta manera, se sugiere que el Gobierno de Colombia debe legislar para impulsar otros sectores, por ejemplo el de servicios públicos de agua potable, con el objetivo de mejorar la cobertura y cumplir así las Metas del Milenio. El buen clima para desarrollar APPs en Colombia se evidencia en el creciente número de proyectos registrados, lo cual se evidencia en la publicación "Infrascope 2012" donde lo ubica en el quinto puesto en Latinoamérica y el Caribe, donde resalta que mejoró su marco normativo limitando las posibilidades de renegociación, además estandarizando los procesos de concesión (The Economist Intelligent Unit, 2013). La importancia de las APPs en el desarrollo de infraestructura en Colombia se fundamenta en:

- Existen recursos disponibles por los fondos de pensiones e inversionistas extranjeros dispuestos a invertir en proyectos de infraestructura.

- Ampliar la base de inversionistas y desarrollo del mercado de capitales.

- Falta de recursos públicos para desarrollar los proyectos.

- Se requiere una adecuada infraestructura para facilitar los tratados de libre comercio.

- Atracción de inversionistas de largo plazo en la provisión de infraestructura pública.

- El mejoramiento de la infraestructura permite al sector privado operar con máximos rendimientos.

- Empresas de países desarrollados quieren invertir en países en vía de desarrollo.

- Correlación entre crecimiento económico y calidad de infraestructura.

\section{Situación actual y tendencias en finan- ciación}

En los procesos de financiación de proyectos de APPs desarrollados por medio del $P F$, el alcance de las entidades financieras no se limita a prestar recursos financieros; al contrario, cada vez tienen más importancia las tareas de asesoramiento financiero (González, 2006), actúan como prestamistas y también como asesores aportando conocimiento y experiencia. Respecto a la estructura de capital, en los proyectos investigados por Esty (2000), el porcentaje de deuda se encuentra entre $65 \%$ al $90 \%$ comparado con el $25 \%$ al $35 \%$ de las empresas típicas de la industria. En este sentido, Hoon et al. (2009) afirman que las estructuras de capital de la mayoría de los proyectos de APPs desarrollados por medio del $P F$ tienen alto grado de apalancamiento, confinanciación de capital quecubren entre 
el $10 \%$ y el $30 \%$ del coste total del proyecto y deuda entre el $90 \%$ al 70\%. Así, Akintoye y Beck (2009) afirman que "las APPs suelen financiarse mediante una combinación de capital y deuda con diferentes proporciones, generalmente la financiación de la deuda supera el $70 \%$, alcanzando en ocasiones el 100\%". En la Figura 5 se muestra en el periodo 2009-2013, 939 proyectos de APPs, con cierre financiero, desarrollados a nivel mundial por medio de $P F$ de los cuales 287 tienen un apalancamiento del $100 \%$, lo cual confirma las evidencias empíricas y la importancia del endeudamiento para el desarrollo de proyectos.

En la Figura 6 se muestra las posibles fuentes de financiación y los porcentajes típicos de financiación de las APPs incluyendo fondos propios, deuda, capital de desarrollo $\mathrm{y}$ financiación intermedia entre deuda y fondos propios (mezzanine); y en la Figura 7 se muestra un resumen de los proyectos APPs desarrollados a nivel mundial por medio de PF en el periodo 2009-2013, donde se confirman dos características importantes en este tipo de proyectos: alto apalancamiento y contratos de largo plazo.

Respecto a la deuda mezzanine, también llamada financiación entresuelo, tiene forma de deuda subordinada porque sus pagos están condicionados al pago de la deuda principal, esto hace que sea más arriesgado que la deuda principal pero menos que los recursos propios invertidos por los promotores; de esta manera el pago se realiza luego de pagar la deuda principal y antes de repartir dividendos (Mascareñas, 2011; Gatti, 2013). Estos recursos pueden ser proporcionados por promotores, inversionistas institucionales, fondos especializados e inclusive

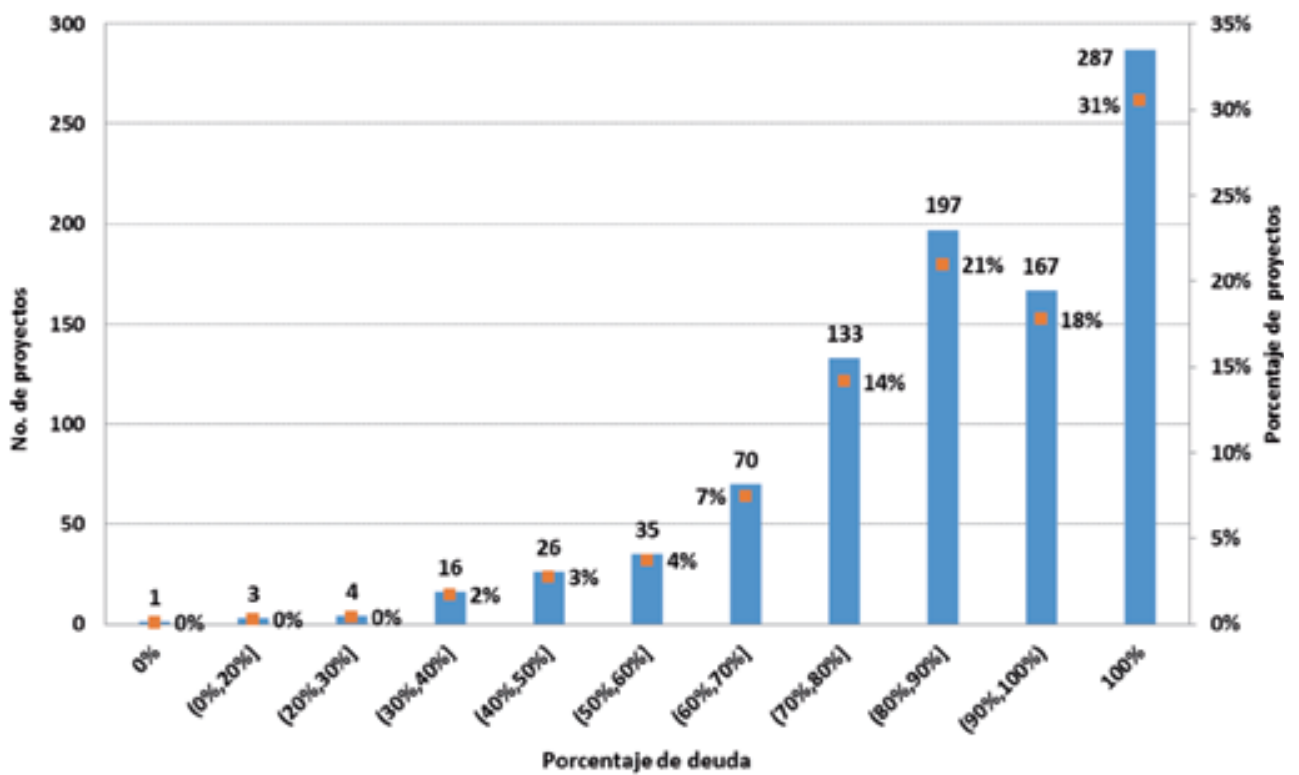

Figura 5: Porcentaje de financiación y número de proyectos APP desarrollados por medio de $P F$ entre 2009 y 2013 , basado en datos de Infrastructure Journal and Project Finance Magazine (2014)

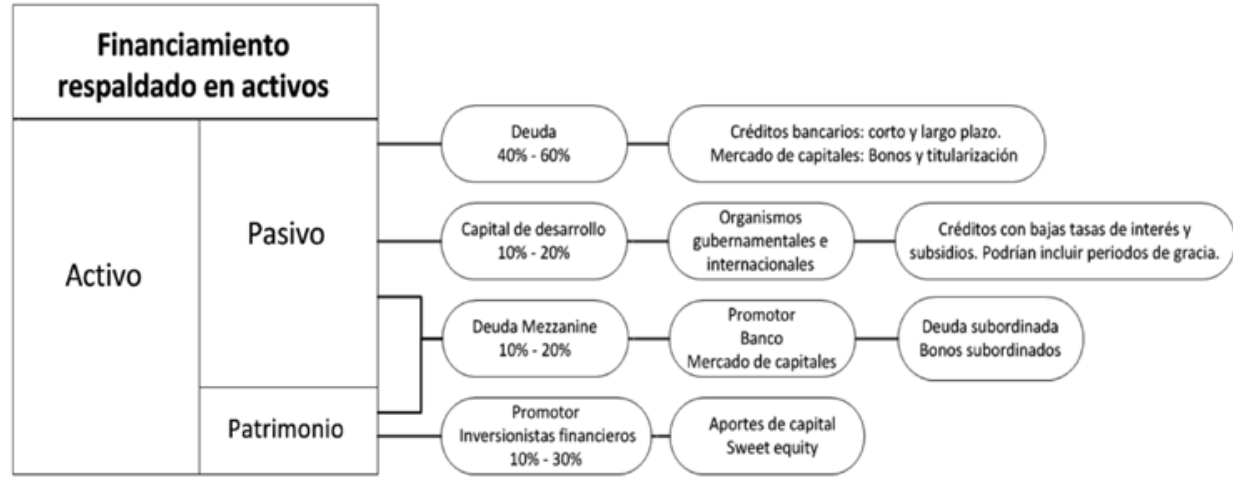

Figura 6: Fuentes de financiación de las APPs, basado en Svědík y Tetřevová (2012) 


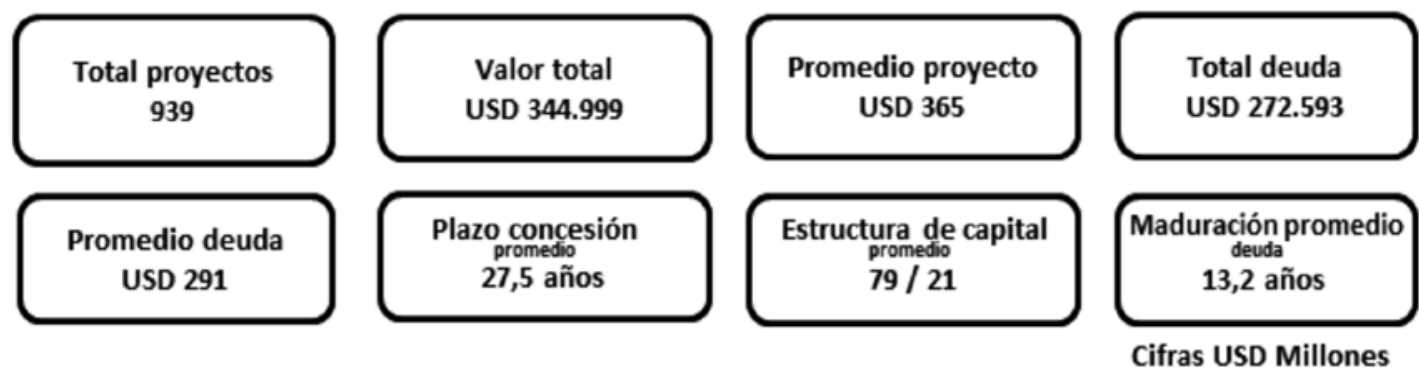

Figura 7: Resumen de proyectos APPs desarrollados por medio de PF en el periodo 2009-2013, basado en datos de Infrastructure Journal and Project Finance Magazine (2014)

organismos multilaterales (World Bank, 2014). Aunque son utilizados en otras formas de finanzas estructuradas no han sido usadas ampliamente en $P F$ (Yescombe, 2007), lo cual abre un camino por explorar y encontrar estructuras que permitan diversificar las fuentes de fondo de los proyectos de infraestructura. Para Colombia, se convierte en una alternativa de financiación la cual deberá estar acompañado del desarrollo del mercado de capitales y el ingreso de inversionistas especializados, migrando así hacia nuevas formas de financiación.

\section{Gestión de riesgos en PF}

Demirag et al. (2011) argumentan que uno de los principales objetivos de los vehículos de inversión es reducir al mínimo los riesgos por medio de la cobertura con seguros y la transferencia a los subcontratistas, éstas son las estrategias más importantes para la gestión y mitigación de riesgos que utilizan las instituciones financieras participantes en transacciones que incluya $P F$ y APPs (ver también Dixon et al., 2005; Akintoye y Chinyio, 2005). Para lograr el éxito requerido en el $P F$, los riesgos del proyecto deben estar identificados y asignados entre las partes de manera adecuada, cuando esto no ocurre la ejecución resulta ineficiente; por esto es necesario que se asignen a la parte mejor preparada para administrarlos (Albújar, 2010). Para Mascareñas (1999), en el $P F$ es esencial definir una metodología para sistematizar el estudio de riesgos, para lo cual propone:

1. Identificar los riesgos inherentes al proyecto o externos al mismo

2. Identificar alternativas de cobertura de riesgo

3. Y elaborar una matriz de riesgos donde se cuantifique el impacto potencial de los mismos y se determine las acciones a seguir
Para identificar los riesgos a los cuales están expuestos los proyectos de infraestructura, en la Tabla 3 se muestran según la etapa: planificación y diseño, construcción y explotación (operación). Como se observa en la Tabla 3, los proyectos independiente de la estrategia de financiación (Corporate Finance o PF), se enfrentan a una gran cantidad de riesgos; así la identificación, atribución y mitigación es indispensable para minimizar la probabilidad de fracaso y por ende no cumplir con las expectativas generadas por los promotores y/o financiadores. Respecto a los principales mecanismos de mitigación de riesgos Rozas et al. (2012) sugieren, por ejemplo, que el riesgo comercial o de demanda puede mitigarse con esquemas de garantía, donde el diseño e implementación aseguren el repago a las entidades bancarias, de manera que pueda reducir los costos financieros. Por otra parte, Aoust et al. (2000) recomiendan las siguientes asignaciones de los principales riesgos de acuerdo a la etapa.

\section{Construcción}

Sobrecosto y retraso: el promotor debe solicitar apoyo a expertos externos para llevar a cabo las tareas de supervisión y fiscalización de los recursos basados en el alcance de la infraestructura. Además garantizar los préstamos durante esta etapa y financiar los sobrecostos vía aumento de capital (aporte de recursos propios) o financiamiento temporal por los accionistas bajo la forma de deuda subordinada. De igual manera los prestamistas pueden extender la financiación otorgando líneas de préstamos contingentes que respeten el equilibro entre el capital y la deuda prevista desde el principio.

Contrapartida: depende la calidad financiera del constructor y se puede cubrir con la emisión de fianzas de ejecución del contrato (performance bonds), cubriendo las penalidades 
Tabla 3: Etapas y riesgos en proyectos de infraestructura, basado en Gatti (2013), Rebeiz (2012), Yescombe (2007), González (2006), Mascareñas (1999), Farquharson et al. (2011) y ANI (2012)

\begin{tabular}{|c|c|c|}
\hline Preparación y Preconstrucción & Construcción & Operación \\
\hline $\begin{array}{l}\text { Político } \\
\text { Financiación } \\
\text { Infraestructura } \\
\text { Corrupción } \\
\text { Proponente } \\
\text { Medioambientales } \\
\text { Diseño } \\
\text { Fiscales } \\
\text { Viabilidad técnica y financiera } \\
\text { Predial } \\
\text { Legal } \\
\text { Cierre financiero }\end{array}$ & $\begin{array}{l}\text { Inflación } \\
\text { Político } \\
\text { Financiación } \\
\text { Disponibilidad de materias primas o mano de obra } \\
\text { Quiebra de la compañía constructora } \\
\text { Retraso, sobrecosto } \\
\text { Mayor costo por remuneración al personal } \\
\text { Disponibilidad del personal idóneo } \\
\text { Medioambientales } \\
\text { Expropiación } \\
\text { Financiero: tipo de interés, tipo de cambio, cierre } \\
\text { financiero o insolvencia del contratista } \\
\text { Arqueológico } \\
\text { Sociales } \\
\text { Infraestructura complementaria } \\
\text { Social } \\
\text { País } \\
\text { Legal } \\
\text { Falta de calidad en las obras } \\
\text { Escasez de cualquier tipo de material y/o insumos } \\
\text { Aseguramiento } \\
\text { Terminación anticipada } \\
\text { Firmas y garantías } \\
\text { Geológico, climatológico y/o hidrológico } \\
\text { Redes de servicios públicos }\end{array}$ & $\begin{array}{l}\text { Político } \\
\text { Financiación } \\
\text { Expropiación } \\
\text { Corrupción } \\
\text { Tarifario } \\
\text { Legal } \\
\text { País } \\
\text { Financiero: Tipo de interés, tipo de } \\
\text { cambio y de liquidez, alteración de las } \\
\text { condiciones de financiación } \\
\text { Disponibilidad del personal idóneo } \\
\text { Administración: excesivo control por } \\
\text { los financiadores. } \\
\text { Medioambientales } \\
\text { Desastres naturales } \\
\text { Terminación de contrato } \\
\text { Demanda } \\
\text { Insuficiencia de suministro } \\
\text { Ineficacia en el equipo de dirección } \\
\text { Social } \\
\text { Mayores cantidades de obra o } \\
\text { variación de precios en insumos } \\
\text { para actividades de operación y } \\
\text { mantenimiento } \\
\text { Aseguramiento } \\
\text { Terminación anticipada } \\
\text { Geológico, climatológico y/o } \\
\text { hidrológico }\end{array}$ \\
\hline
\end{tabular}

que se pueden presentar debido a retrasos o sobrecostos imputables al constructor.

Tasa de interés: Puede cubrirse con una línea contingente establecida por los accionistas y prestamistas o por medio de coberturas con swaps de tipos de interés of forward de divisas.

\section{Operación}

Demanda: teniendo en cuenta los factores propios de cada proyecto, este riesgo se podría distribuir entre los accionistas, el operador y el Estado, éste último podría asegurar un adecuado nivel de tráfico o garantizar acuerdos de compra en firme por medio de contratos take or pay u offtakes, los cuales son utilizados en proyectos de energía y agua.

Aumento de costos de operación: en primera instancia podrían asignarse al consorcio si el aumento del costo es responsabilidad del operador o concesionario, de esta manera no se sugiere asignarla a los usuarios dado que un aumento podría representar un obstáculo para el desarrollo de la APPs. En el caso que ocurra por un origen externo, se sugieren las fórmulas de subsidio otorgadas por la autoridad competente. 


\section{Riesgos indirectos permanentes en el entorno}

Fuerza mayor: se deben aportar las mismas cláusulas de fuerza mayor en los diferentes contratos, esto no significa que el riesgo desaparece, pero hace posible establecer negociaciones que permitan encontrar soluciones, considerando que no es posible transferir todo el riesgo al sector privado.

Jurídicos: es necesario incluir en los contratos cláusulas de estabilidad del marco legal y reglamentario, así como posibilidad de renegociación o de reglamentación.

De acuerdo a lo anterior, si las cláusulas contractuales no están bien escritas (o los riesgos no están bien asignados) se reducirán los beneficios y la incidencia de los conflictos evidenciados en renegociaciones se incrementarán significativamente. Por tanto, un Estado sólido con políticas legales y fiscales estables son determinantes en la integralidad contractual (Guasch, 2014), lo cual es confirmado por Fay y Morrison (2006), indicando que "las renegociaciones pueden ser también consecuencia de contratos mal concebidos, de la debilidad de los reglamentos o de ambos factores, donde el mejor diseño de las concesiones desalentaría las renegociaciones innecesarias". Para Colombia, el artículo 35 del decreto 1467 de 2012 el cual regula la Ley de APPs 1508 de 2012, establece que será la autoridad estatal competente la responsable de tipificar, estimar y asignar los riesgos asociados en los proyectos de APPs (Congreso de Colombia, 2012b), lo cual dependerá de las características propias del proyecto. Una vez identificados los principales riesgos, se debe realizar una matriz de riesgos que resuma las variables y su riesgo asociado, así como las estrategias o alternativas que podrían seguir en cada caso (Mascareñas, 1999). Adicionalmente Arias (2012) indica las principales características de los riesgos asociados en un PF (Tabla 4).

Bracey et al. (2006) indican que en los acuerdos para el desarrollo de proyectos, el sector público transfiere riesgos al sector privado por la gestión más eficiente que éste realiza, dado que tiene un incentivo para gestionarlos de manera eficiente e innovadora. Sin embargo, existe la tendencia natural del Estado en trasladar la mayor cantidad de riesgos al vehículo de inversión; por tanto, es necesario que exista un equilibrio en la repartición de riesgos para no atentar contra la viabilidad del proyecto (Albújar, 2010). Por su parte Jin et al. (2011) indican que compartir los riesgos en lugar de transferirlos y buscar un beneficio mutuo deben ser las principales características.

Tabla 4: Principales características de los riesgos asociados en un Project Finance, basado en Arias (2012)

\begin{tabular}{|l|l|}
\hline Riesgo & \multicolumn{1}{|c|}{ Principal característica } \\
\hline Físicos & $\begin{array}{l}\text { Aquellos fenómenos que afectan la } \\
\text { construcción y explotación del proyecto }\end{array}$ \\
\hline Tecnológicos & $\begin{array}{l}\text { Cuando el uso de cierto instrumento afecta } \\
\text { e impide la máxima producción del bien } \\
\text { objeto del proyecto }\end{array}$ \\
\hline De construcción & $\begin{array}{l}\text { Demoras o incumplimientos en la fase de } \\
\text { construcción y en consecuencia posterga } \\
\text { la operación del proyecto. }\end{array}$ \\
\hline De & $\begin{array}{l}\text { Es posible que las condiciones del } \\
\text { contrato de concesión y de la licencia } \\
\text { cambien, dándole a la entidad estatal el } \\
\text { derecho a terminar la relación contractual } \\
\text { o revocarla en términos inicialmente } \\
\text { pactados. }\end{array}$ \\
\hline Ambientales & $\begin{array}{l}\text { Daños potenciales o actuales que la } \\
\text { construcción y explotación del proyecto } \\
\text { genere para el medio ambiente. }\end{array}$ \\
\hline $\begin{array}{l}\text { Asociado con la inestabilidad del } \\
\text { gobierno o del Estado, y corresponde a } \\
\text { la posibilidad que las condiciones con las } \\
\text { cuales se otorgó la licencia o se celebró } \\
\text { el contrato se afecten por un cambio } \\
\text { de políticas del Estado o por cambio de } \\
\text { gobierno }\end{array}$ \\
\hline Pón
\end{tabular} \mid

\section{Mecanismos de control usando PF}

La importancia de los mecanismos de control y seguimiento por parte de los promotores, financiadores y el Estado, han llevado a utilizar indicadores que midan la capacidad del proyecto para pagar el servicio a la deuda y el cumplimiento de niveles de calidad y servicio de la infraestructura. Los principales mecanismos utilizados, en la práctica, por las instituciones financieras son la Razón de Cobertura del Servicio de la Deuda RCSD y la Razón de Cobertura de Vida del Préstamo RCVP (Borgonovoa et al., 2010), los cuales miden la cobertura del flujo de caja libre respecto al servicio de la deuda. Éstos ayudan a evaluar la situación de impago, para lo cual Aragonés et al. (2009) afirman que en el $P F$ la situación de impago sucede cuando determinados 
indicadores que relacionan el pago de la deuda y los flujos del proyecto se encuentran por debajo de un determinado nivel, mientras que en modelos de evaluación de riesgo de crédito, como el KMV (Kecholfer, McQuown y Vasicek), el cual se basa en la teoría de opciones, se produce cuando el valor de los activos se sitúa por debajo del valor de la deuda. En el seguimiento al proyecto, para validar el cumplimiento de los indicadores o condiciones básicas del proyecto, se utilizan las cuentas auditadas del vehículo de inversión, de forma que las entidades financieras podrían exigir la expedición de certificados (López y García, 2005). En la Tabla 5 se presentan las RCSD y RCVP promedio utilizado en diferentes sectores (Gatti, 2013).

Tabla 5: RCSD y RCVP promedio por sectores (Gatti, 2013)

\begin{tabular}{|l|c|c|}
\hline Sector & $\begin{array}{c}\text { RCSD } \\
\text { promedio }\end{array}$ & $\begin{array}{c}\text { RCVP } \\
\text { promedio }\end{array}$ \\
\hline Energía & & \\
• Productores independientes & $2.00 \mathrm{x}-2.25 \mathrm{x}$ & $2.25 \mathrm{x}-2.75 \mathrm{x}$ \\
- Con acuerdo de pago & $1.50 \mathrm{x}-1.70 \mathrm{x}$ & $1.50 \mathrm{x}-1.80 \mathrm{x}$ \\
- Mercados regulados & $1.40 \mathrm{x}-1.45 \mathrm{x}$ & $1.40 \mathrm{x}-1.45 \mathrm{x}$ \\
Transporte & $1.35 \mathrm{x}-1.50 \mathrm{x}$ & $1.40 \mathrm{x}-1.60 \mathrm{x}$ \\
Telecomunicaciones & $1.35 \mathrm{x}-1.50 \mathrm{x}$ & $1.35 \mathrm{x}-1.50 \mathrm{x}$ \\
Agua & $1.20 \mathrm{x}-1.30 \mathrm{x}$ & $1.30 \mathrm{x}-1.40 \mathrm{x}$ \\
\hline
\end{tabular}

Otros indicadores identificados en la literatura y utilizados en analizar la viabilidad financiera de proyectos de infraestructura son: valor presente neto, valor presente neto ajustado, tasa interna de retorno, tasa interna de retorno modificada, capacidad de autofinanciamiento, ratio de recursos propios en proyectos de riesgo (Iyer y Sagheer, 2012), y el valor presente neto desarticulado (decoupled net present value) (Espinoza y Morris, 2013). Por otra parte, el Estado requiere medir las condiciones pactadas respecto a la disponibilidad y calidad de la infraestructura, para ello determina de acuerdo a las características del proyecto y requerimientos de uso, indicadores que permitan monitorear y controlar las condiciones previamente acordadas (Yescombe, 2007), que garanticen la prestación del servicio con eficacia.

Morin (2014) argumenta que el diseño de buenos indicadores dependerá de la claridad que se tenga sobre lo que se quiere medir y éstos pueden estar en función de diferentes aspectos, tales como: disponibilidad, limpieza, número de estudiantes, nivel de tráfico, pacientes atendidos, número de ambulancias por kilómetro, entre otros, los cuales dependerán del tipo de proyecto.

Para Colombia, los indicadores son definidos por la entidad estatal competente con el objetivo de medir el cumplimiento de la disponibilidad, estándares de calidad y nivel de servicio, los cuales previo cumplimiento de lo pactado tendrían el derecho a retribuciones por parte del Estado. De igual manera existirá un mecanismo de deducciones graduales en caso de no cumplirse lo pactado, los cuales estarán expresados en el contrato (Congreso de Colombia, 2012b). Estas retribuciones son llamadas Vigencias Futuras y representan las partidas que destina el Estado para el desarrollo de proyectos de infraestructura y que se entregarán previo cumplimiento de lo pactado en el contrato, estos recursos pueden ser como máximo el $30 \%$ en dólares, permitiendo al SPV diversificar las fuentes de fondo y así obtener créditos en dólares teniendo una cobertura natural respecto al tipo de cambio. Así mismo, para hacer seguimiento respecto a los niveles de servicio en los proyectos de infraestructura, Liu et al. (2014) proponen un sistema de gestión del desempeño (Performance Management Systems PMS), el cual consiste en una serie de indicadores basados en el ciclo de vida del proyecto, ofreciendo herramientas necesarias para tener el control del rendimiento en tiempo real, lo cual conlleva a la mejora de la calidad en el servicio.

\section{APPs: participación privada en infraestructura pública}

Para Grimsey et al. (2005), las APPs llenan el espacio entre los proyectos tradicionalmente realizados por el Estado y la privatización; además, afirman que no existe una definición única de APPs; según el país, el término puede cubrir una variedad de transacciones en las cuales el sector privado tiene el derecho de explotar por un período prolongado un servicio tradicionalmente de responsabilidad del sector público, incluyendo desde contratos de gestión a corto plazo (con poca o ninguna inversión de capital) o de concesión, que pueden incluir diseño y construcción, junto con la prestación de una serie de servicios o la financiación de la totalidad de la construcción y operación. La Tabla 6 presenta los principales aportes a la definición de APPs.

Las APPs denotan una interfaz moderna entre las autoridades públicas y las privadas, dado que tienen por objeto la ejecución de proyectos de infraestructura o de servicios públicos (Bovis, 2010), los cuales tienen 
usualmente un plazo entre 20 y 30 años (Engel et al., 2013). Tang et al. (2010) argumentan que las cuestiones culturales y políticas también deben abordarse en las APPs, dado que no pueden ser simplemente copia de un país a otro; adicionalmente, los entornos políticos, jurídicos y económicos apropiados son esenciales para las APPs; condición para desarrollarlas sin problemas.

\section{Principales esquemas de APPs}

De acuerdo con Vasallo et al. (2010) y Hoon et al. (2009), los esquemas de APPs se fundamentan en la asignación al sector privado de la prestación y/o provisión de diferentes servicios e infraestructura, los cuales se definen de acuerdo al alcance de sus funciones, en la Tabla 7 se describen los principales esquemas utilizados; en el nombre del esquema se indica el acrónimo en inglés, los cuales son usados ampliamente.

Para Farquharson et al. (2011), las APPs son algunas veces confundidas con privatización; sin embargo, la privatización implica la permanente transferencia de un activo público al sector privado, mientras que las APPs implican una relación continua con el sector privado. Así, el motivo por el cual el sector privado comienza a participar en la provisión de infraestructura pública, se fundamenta en las limitaciones de endeudamiento del sector público, que no permite que la administración afronte todos los proyectos de inversión; el Estado no afronta la inversión directamente, sino que cede la construcción y explotación al sector privado, que recupera la inversión por medio de la explotación (González, 2006).

Tabla 6: Principales aportes a la definición de APP

\begin{tabular}{|l|l|}
\hline Referencia & \multicolumn{1}{c|}{ Definición } \\
\hline Pöll (2012) & $\begin{array}{l}\text { Las APPs (Public-Private Partnerships) no es un modelo de financiación, sino un modelo } \\
\text { contractual y de organización de largo plazo, entre el sector público y privado para la provisión de } \\
\text { infraestructura, además de bienes y servicios. }\end{array}$ \\
\hline Grimsey y Lewis (2002) & $\begin{array}{l}\text { Acuerdo a largo plazo entre organismos de los sectores público y privado para la gestión de } \\
\text { instalaciones de infraestructura }\end{array}$ \\
\hline Banco Mundial (2014) & $\begin{array}{l}\text { Afirma que no existe una definición aceptada de APPs; sin embargo, indica que hace referencia } \\
\text { al acuerdo entre el sector público y privado, donde parte de los servicios o labores que son } \\
\text { responsabilidad del sector público son suministrados por el privado, bajo un acuerdo de objetivos } \\
\text { compartidos para el abastecimiento del servicio público o de infraestructura. }\end{array}$ \\
\hline $\begin{array}{l}\text { Congreso de Colombia } \\
\text { (2012a) }\end{array}$ & $\begin{array}{l}\text { Instrumento de vinculación de capital privado, que se materializa en un contrato entre una entidad } \\
\text { estatal y una persona natural o jurídica de derecho privado, para la previsión de bienes públicos } \\
\text { y de sus servicios relacionados, que involucra la retención y transferencia de riesgos entre las } \\
\text { partes y mecanismos de pago, relacionados con la disponibilidad y el nivel de servicio de la } \\
\text { infraestructura y/o servicio. }\end{array}$ \\
\hline $\begin{array}{l}\text { Comisión de las } \\
\text { Eomunidades }\end{array}$ & $\begin{array}{l}\text { Formas de cooperación entre las autoridades públicas y el mundo empresarial, cuyo objetivo es } \\
\text { garantizar la financiación, construcción, renovación, gestión o mantenimiento de infraestructura, } \\
\text { o la prestación de un servicio. }\end{array}$ \\
\hline $\begin{array}{l}\text { Federal Highway } \\
\text { Administration (2007) }\end{array}$ & $\begin{array}{l}\text { Acuerdo contractual formado entre el sector público y socios del sector privado. Los acuerdos por } \\
\text { lo general implican para una agencia del gobierno la contratación con una empresa privada para } \\
\text { renovar, construir, operar, mantener y/o gestionar una instalación o sistema. Mientras el sector } \\
\text { público por lo general conserva la propiedad de la instalación o red, la parte privada se le dará } \\
\text { derechos de decisión adicionales para determinar cómo se completará el proyecto o tarea. }\end{array}$ \\
\hline
\end{tabular}


Tabla 7: Principales esquemas de APP (Vasallo e Izquierdo, 2010; Hoon et al., 2009)

\begin{tabular}{|l|l|}
\hline Esquema & \multicolumn{1}{|c|}{ Descripción } \\
\hline Operación y mantenimiento (OM) & $\begin{array}{l}\text { El sector privado es responsable de todos los aspectos de operación y mantenimiento de la } \\
\text { infraestructura. }\end{array}$ \\
\hline $\begin{array}{l}\text { Construir, operar y transferir } \\
\text { (BOT) }\end{array}$ & $\begin{array}{l}\text { El sector privado financia y construye la infraestructura, y explota el activo durante un período } \\
\text { de tiempo, fijo o variable, acordado contractualmente, para luego transferirlo al Estado. Este es } \\
\text { el mecanismo que más se utiliza en las concesiones de infraestructura pública financiadas por } \\
\text { medio de la técnica de PF. }\end{array}$ \\
\hline $\begin{array}{l}\text { Construir, propietario, operar y } \\
\text { transferir (BOOT) }\end{array}$ & $\begin{array}{l}\text { Se diferencia del esquema anterior porque la propiedad del activo lo tiene el consorcio privado } \\
\text { que lo explota, hasta el momento que lo transfiere al Estado. En algunos países la legislación } \\
\text { considera que la infraestructura de interés general es pública, por lo que impone que los } \\
\text { activos sigan siendo propiedad de la administración, aunque se encuentren fuera del balance. } \\
\text { Lo anterior impide que jurídicamente se pueda aplicar este esquema. }\end{array}$ \\
\hline $\begin{array}{l}\text { Construir, propietario, operar } \\
\text { (BOO) }\end{array}$ & $\begin{array}{l}\text { A diferencia del anterior, no hay transferencia de los activos al Estado. Este esquema se } \\
\text { aproxima a la privatización del activo. }\end{array}$ \\
\hline $\begin{array}{l}\text { Diseñar, construir, financiar y } \\
\text { operar (DBFO) }\end{array}$ & $\begin{array}{l}\text { El nombre proviene del Reino Unido dentro de la Private Finance Initiative PFI, es parecido al } \\
\text { segundo esquema, con la diferencia que el sector privado asume un rol más importante en el } \\
\text { diseño. La propiedad de los activos permanece en la administración pública, por lo que debe } \\
\text { remunerar al consorcio privado encargado de explotar el proyecto, habitualmente por medio } \\
\text { del "peaje sombra". }\end{array}$ \\
\hline
\end{tabular}

\section{Principales ventajas y desventajas de las APPS}

Una de las principales ventajas que justifica la elección de APPs es la agrupación o empaquetamiento de actividades entre los diferentes participantes, lo cual puede mejorar la eficiencia productiva (Engel et al., 2010); permitiendo la expansión y mejora de la red de relaciones entre los participantes, con resultados como: economías de escala, reducción de costos de transacción, diversificación de riesgos y flexibilidad en el diseño del proyecto (Zhang et al., 2013). Asimismo, Gómez et al. (2001) consideran que las ventajas para el sector público en el uso de APPs son:

- Agilizar el proceso de desarrollo de infraestructura

- Permite dirigir los fondos públicos hacia necesidades sociales, donde el sector privado no invertiría

- Transferir riesgos al sector privado

- Permitir, de forma políticamente aceptable, transformar la práctica de financiación vía impuestos, en financiación directa del usuario

Las ventajas de las APPs se fundamentan en los incentivos que tiene el sector privado para generar eficiencia en el proyecto, para lo cual el Estado podría exigir índices de servicio que deberá cumplir; en caso contrario, se podrían aplicar las penalidades descritas en los contratos (Bravo, 2011), y así el Estado puede concentrase en sus competencias básicas (Tang et al., 2010). Esta ventaja se convierte en peligro cuando una empresa sin experiencia previa en proyectos similares recibe un contrato de APPs (Mouraviev y Kakabadse, 2012). Por otra parte, Hodge y Greve (2005) en la revisión crítica del funcionamiento y resultados en las APPs, indican que de acuerdo a los hallazgos presentados en evidencias empíricas, los costos y beneficios son limitados y mixtos; argumentan además, que las ventajas financieras y económicas son todavía objeto de debate. En consecuencia, las renegociaciones son objeto de estudio por las consecuencias derivadas de una deficiente planeación y estructuración de los proyectos, lo cual podría impactar los resultados esperados.

Para Xion y Zhang (2014) las renegociaciones son muy comunes en las APPs, y en la mayoría de las ocasiones los gobiernos indemnizan a los promotores por pérdidas; ellos consideran que los modelos más populares en proyectos 
de infraestructura vial como mecanismos de renegociación son: ajuste del peaje, extensión del contrato y el subsidio anual o ajuste de pago. Además concluyen que el núcleo de los modelos de renegociación se basa en el análisis de flujo de caja, la previsión de la futura demanda de tráfico y los costes de operación y mantenimiento. En los estudios de casos realizado por Cruz et al. (2014), se demostró que el sector privado, por lo general, es el primero en iniciar un proceso de renegociaciones. Esto se debe a las expectativas no satisfechas, como por ejemplo previsiones de tráfico o la incapacidad del gobierno para cumplir con las obligaciones contractuales, como la obtención de permisos ambientales.

Respecto a las indemnizaciones por rescisión anticipada en las APPs, en especial de servicios públicos, Xiong et al. (2014) proponen dos enfoques basados en estrategias para mitigar el riesgo presentado en proyectos de largo plazo donde existe incertidumbre asociada en la demanda. El primero calcula la compensación basada en el costo y los ingresos acumulados antes de la fecha de terminación anticipada; y el segundo, en el valor de los flujos de caja descontados del tiempo que falta para terminar el contrato, los cuales tienen alta incertidumbre.

\section{Renegociaciones en Latinoamérica: caso Colombia}

Debido a la cantidad de contratos que conlleva una APPs y las consecuencias derivadas de seleccionar un esquema inadecuado, o no haber asignado de manera eficiente los riesgos y los beneficios, se podrían presentar renegociaciones en los contratos. Por ejemplo, para Vives et al. (2007) en Latinoamérica durante los años noventa el $40 \%$ de 796 contratos de concesiones de infraestructura fue renegociado, con un tiempo aproximado de renegociación de 2.2 años, lo cual es reafirmado por Guasch (2004), indicando que la alta incidencia de las renegociaciones debería ser motivo de preocupación, en vista de los costos agregados y las importantes pérdidas irrecuperables perjudicando el bienestar general.

Por otra parte, Rozas et al. (2012) consideran que las renegociaciones podrían convertirse en un instrumento positivo y necesario cuando se refiere a algún aspecto no incluido originalmente en el contrato o cambios no previstos en las condiciones pactadas. Adicionalmente, Fay y Morrison (2006) indican que puede ser deseable tener algún nivel de renegociación para remediar los problemas que susciten y mejoren el diseño de los contratos; sin embargo, el alto número de renegociaciones en Latinoamérica refleja la mentalidad de incumplimiento y un comportamiento abusivo, tanto del sector privado como del público, indicando que los operadores actúan de forma oportunista o los contratos se diseñan mal. Asimismo, Guasch (2014) considera que el desafío en las renegociaciones es mejorar el valor para todas las partes, incluidos los usuarios de los servicios, considerando además las posibles consecuencias fiscales por la modificación del contrato.

De acuerdo con Rozas et al. (2012), en la literatura especializada se señala el exceso de renegociaciones como uno de los principales problemas en el desarrollo de infraestructura, en la mayoría de los casos se asocia a la insuficiente preparación de estudios de demanda y costos, donde se pueda definir los estándares de calidad, los costos de inversión y operación, así como los plazos de recuperación de la inversión conforme a las tarifas establecidas.

De la misma manera, Fay y Morrison (2006) consideran que las renegociaciones son costosas, presentan desventajas financieras, perturban los servicios y disminuyen la credibilidad del modelo de las APPs, resaltando que cualquiera que sea la causa, las renegociaciones frecuentes soncostosas. Enconsecuencia, para Guasch y Spiller(2001). éstas elevan el costo de capital porque los inversionistas exigirán una prima de riesgo en compensación del peligro que cambien las normas y la reglamentación, estimándola en un rango entre $2 \%$ y $6 \%$ según el país y sector.

Para el caso de Colombia, en el periodo 1993 a 2010 se presentaron 430 renegociaciones en 25 concesiones de carreteras, cifra superior comparándola con Chile y Perú que tuvieron 60 y 53 renegociaciones en 21 y 19 concesiones respectivamente (Guasch et al., 2014). Adicionalmente, de acuerdo con Fay y Morrison (2006), un efecto de las renegociaciones en Colombia se evidencia en los enormes pasivos contingentes derivados de las garantías por parte del Estado, por ejemplo en niveles de demanda o de tipo de cambio, en las APPs (inicialmente llamadas Concesiones bajo la Ley 80), representaron según estimaciones el $4 \%$ del Producto Interno Bruto. De esta manera se considera que en muchos países de Latinoamérica se necesita fortalecer los procesos de elaboración y licitación de los contratos, con 
el objetivo de proteger los acuerdos inicialmente pactados junto con el establecimiento de mecanismos adecuados de conciliación y arbitraje para resolver las posibles disputas. (Rozas et al., 2012).

Por otro lado, siempre existirá una diferencia entre los valores estimados y reales, por esta razón la ley colombiana incorpora el concepto de Equilibrio Económico del Contrato, el cual establece que los contratos pueden ser renegociados cuando existan situaciones imprevistas que afecten significativamente la posición económica de las partes; sin embargo, para evitar los abusos y el detrimento patrimonial, se establecieron los siguientes límites contemplados en la Ley 1508 de 2012 (Fedesarrollo, 2012):

- Límite al aumento del valor del contrato: el artículo 13 establece que las adiciones o prórrogas no podrán superar el $20 \%$ sobre el valor del contrato originalmente pactado, el cual está determinado por el presupuesto estimado de inversión. En Chile y España corresponde al $15 \%$ y $20 \%$ respectivamente.

- Plazo concesión: el artículo 6 y 13 indican que las prórrogas en tiempo serán valoradas por la entidad estatal correspondiente y no podrán superar el $20 \%$ del valor del contrato ni los 30 años.

- Nivel de servicio: los hitos que requieran obras adicionales por valores superiores al $20 \%$ del presupuesto original, deben implicar un nuevo proceso de licitación. En el caso de Chile, corresponde al 5\%.

- Tiempo de renegociación: el artículo 7 indica que solo se podrá renegociar adiciones o prorrogas después de los primeros tres años de vigencia del contrato y hasta antes de cumplir las primeras tres cuartas (3/4) partes del plazo inicialmente pactado en el contrato.

- Desequilibrio económico: el contrato debe fijar y definir el límite que establezca las renegociaciones con el fin de ajustarlo a los cambios, y en caso de disputas posteriores se deberá establecer un mecanismo eficaz de resolución de conflictos que impida el desarrollo del contrato. Una posible alternativa es el modelo de arbitramiento de la Federación Internacional de Ingenieros Consultores FIDIC.

- Repago de la deuda: en caso que la calidad crediticia se perjudique por acciones del promotor o existan cambios en el objeto del contrato, los tenedores de la deuda podrán exigir el repago acelerado. De igual manera, el artículo 30 establece que en caso de incumplimiento, los financiadores podrán continuar con la ejecución del contrato hasta su terminación.

Por lo anterior, si las condiciones de contratación están debidamente limitadas en términos de porcentajes, precios y oportunidades, y la incertidumbre del proyecto es reducida, aquellos proponentes que oferten precios bajos con el objetivo de renegociar, tendrían que asumir a expensas de sus utilidades, la diferencia entre su oferta y el costo real de la obra (Fedesarrollo, 2012). Así, el uso de APPs para reemplazar o complementar la prestación pública de la infraestructura es común en los últimos años; los gobiernos reducen el endeudamiento público y se benefician de la eficacia de la empresa privada (Meaney y Hope, 2012).

\section{Resultados}

Después de revisar la literatura científica Kayser (2013) y Tang et al. (2010) identifican varias categorías las cuales se podrían agrupar en cuatro áreas esenciales respecto al estado de la cuestión y la evolución de la investigación en APPs y $P F$, éstas ocupan la mayoría de publicaciones y proporcionan ideas relevantes para el diseño de futuros programas de investigación; en la Figura 8 se muestran las categorías agrupadas por áreas.

En esta misma línea, Kleimeier (1995) y Gatti (2013) argumentan que en el campo de las finanzas, la investigación en APPs y $P F$ es todavía muy limitada en la academia, en los desarrollos teóricos y en la aplicación empírica. Así mismo, para resaltar la importancia de investigar acerca de éstos temas, Esty (2004) considera que las razones académicas se basan en la capacidad para realizar investigaciones en un entorno productivo e informativo; indica también que existen pocas publicaciones de trabajos sobre $P F$, así como textos de finanzas corporativas que lo incluyen dentro de las temáticas. Para Gatti (2013) existen cuatro diferentes corrientes relacionadas con el $P F$ en la literatura:

1. Plantea la incorporación óptima de nuevos proyectos dentro del vehículo de inversión.

2. Concibe el $P F$ como un subconjunto del mercado de 


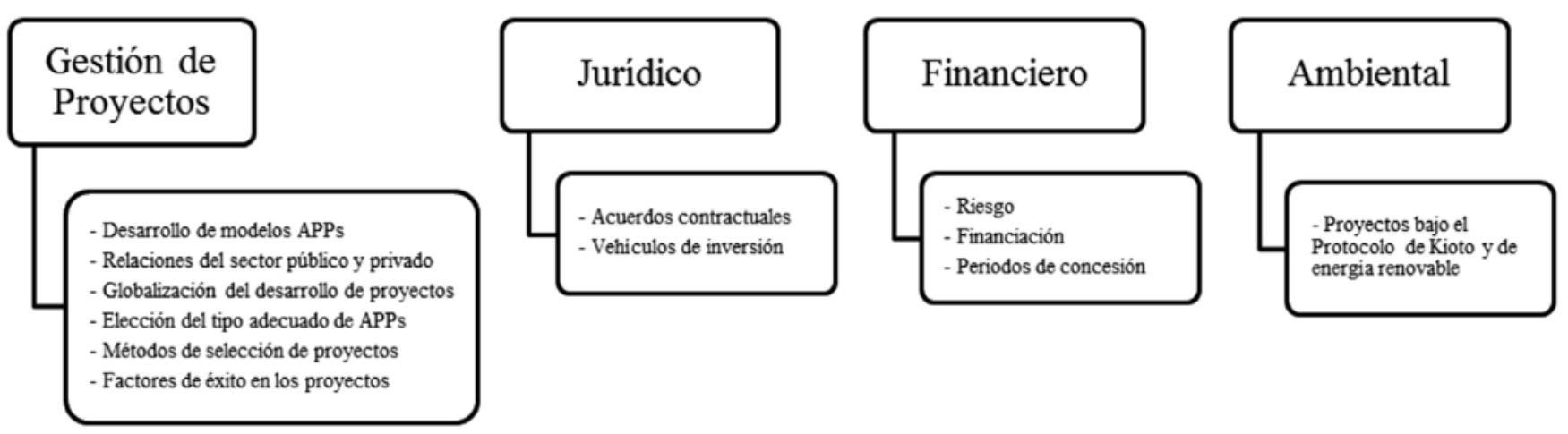

Figura 8: Áreas y categorías de investigación de PF y APPs, basado en Kayser (2013) y Tang et al. (2010)

créditos sindicados.

3. Aborda la relación entre el uso de los contratos financieros, la gestión del riesgo empresarial y la toma de decisiones financieras.

4. Y otra que analiza las APPs en comparación con los mecanismos tradicionales de contratación pública, donde el uso del $P F$ como técnica de financiación es asociado frecuentemente.

Las razones para estudiar el APPs y $P F$ se soportan en el potencial de desarrollo de nuevas teorías financieras, dado que existe un amplio margen en ese campo para realizar investigaciones de tipo descriptivo y cuantitativo, en especial en las economías en desarrollo, donde se presenta un desarrollo exponencial de proyectos de infraestructura (Esty, 2004). Considerando que existe una necesidad en identificar soluciones innovadoras y nuevos enfoques para la financiación y ejecución de las inversiones, ésto puede significar la identificación de nuevas formas de mezclar diferentes instrumentos de financiación con enfoques que ayuden a cerrar la brecha de la viabilidad financiera por medio de la creación de mecanismos más innovadores (World Bank, 2012).

Por tanto, el estudio de grandes proyectos de infraestructura tiene la capacidad de generar una visión académica que contribuya a mejorar la práctica actual (Esty, 2004); donde la identificación exacta del efecto de la eficiencia del $P F$ y las APPs en el sector de infraestructura, ofrece una vía pertinente para futuras investigaciones (Kleimeier et al., 2010). Considerando además que las APPs desde un punto de vista teórico no se han estudiado ampliamente y necesitan una teoría de acuerdos público-privados que está en proceso de construcción (Saussier, 2013), y que a pesar de la creciente importancia internacional del $P F$ para financiar proyectos a gran escala, hay una escasez de estudios en esta área (Girardone y Snaith, 2011). Adicionalmente, Hinojosa (2009) propone futuras investigaciones para desarrollar la definición y valoración de las opciones reales de infraestructura en contratos de largo plazo para APPs. Lo cual es apoyado por Martins et al. (2013), indicando que "las opciones reales surgen debido a la necesidad de un nuevo enfoque para la gestión de infraestructuras y la valoración, ya que el método de flujo de caja descontado no permite captar el valor de la flexibilidad, que preferiblemente debe ser incorporado en cualquier proyecto de infraestructura; así la importancia en el campo de la infraestructura está despertando el interés de muchos gerentes". De acuerdo con Hinojosa (2010), la mayoría de las investigaciones en finanzas corporativas en los últimos 40 años se han centrado en estudiar el teorema de Modigliani y Miller, el cual relaciona bajo ciertas condiciones la irrelevancia de la estructura financiera respecto al valor de la empresa; pero en el caso del $P F$, ésta sí importa, considerando que la deuda y los aportes de capital por parte de los promotores tienen efectos en las decisiones de inversión, generando interés para futuras investigaciones en términos teóricos y prácticos.

Respecto a la teoría de agencia y su aplicación en el $P F$, Jensen y Meckling (1976) y Jensen (1986) argumentan que "a nivel empírico, el $P F$ provee un soporte importante para el estudio de teoría de agente principal en la estructura de capital en presencia de contratos incompletos, como es el caso general de los APPs". Por otra parte, el incumplimiento de las garantías exigidas en los contratos de proyectos de infraestructura y enunciando líneas de investigaciones futuras sobre la estructura financiera del $P F$, Rosillo et 
al. (2012) consideran necesario agregar restricciones en la programación matemática de la estructura financiera, indicando que "las Cadenas de Markov, dado el carácter estacionario de las probabilidades, y la programación dinámica como instrumento optimizador serían elementos importantes que darían más robustez al modelo". Igualmente Cruz et al. (2014) consideran que un aspecto crítico para el éxito de los acuerdos en APPs es la renegociación de los contratos, señalando que se requiere más evidencia empírica para proporcionar a los académicos y profesionales una mejor comprensión de las cuestiones abordadas.

Por último, Kleimeier (1995) indica que el $P F$ es un campo abierto para los investigadores académicos y lo recomienda para cualquier investigador interesado en las finanzas; sugiere además, extender los estudios sobre modelos óptimos de estructura de capital y la comprobación empírica de sus consecuencias e investigaciones sobre los modelos de préstamos.

\section{Comentarios finales}

Se ha determinado la relación entre el $P F$ y las APPs, lo cual pone de manifiesto la importancia en el desarrollo de proyectos de infraestructura, identificando la interacción conjunta para la provisión de infraestructura pública. El primero como técnica de financiación de proyectos, la cual tiene como principio servir de herramienta para la gestión de riesgos, y el segundo como mecanismo de vinculación del sector privado. La importancia de la banca multilateral como uno de los principales financiadores y medio para la gestión del riesgo político deriva en la sindicación de créditos que incentivan la participación de la banca local, lo cual motiva la reducción en el costo de financiación.

Debido a los altos costos de transacción que se presentan en los proyectos desarrollados por medio de $P F$, el no cumplir con las condiciones de disponibilidad y nivel de servicio tendrían un impacto negativo, en términos de rentabilidad. Por tanto, es deber de los desarrolladores de proyectos tener un estricto control de los costos asociados en la estructuración y además cumplir lo pactado con el Estado referente a disponibilidad y nivel de servicio, considerando que si el proyecto tiene aprobado Vigencias Futuras éstas podrían disminuir por el incumplimiento, afectando los recursos proyectados. Igualmente, para el
Estado representa tener los mecanismos y la capacidad institucional necesaria para monitorear el cumplimiento, estableciendo parámetros para el desarrollo de la función social de la infraestructura y la fiscalización de los recursos públicos.

A partir de la revisión de la literatura especializada y la situación actual en Colombia se identifican diferentes líneas de investigación que sirven para promover investigaciones cualitativas y cuantitativas que aumenten el acervo de conocimiento y permitan disminuir la distancia entre la teoría y la práctica, las cuales permitan tener elementos de análisis y discusión de la realidad del desarrollo de proyectos de infraestructura en Colombia:

- Créditos sindicados: evaluar el impacto de la sindicación de créditos en el desarrollo de proyectos de infraestructura

- Comisiones: medir el impacto de las comisiones generadas por la sindicación de créditos y asesores externos en la rentabilidad y éxito del proyecto.

- Vehículo de inversión: caracterizar los Patrimonios Autónomos como vehículos de inversión, indicando el estado del sector fiduciario.

- Derivados: evaluar el uso e impacto de las coberturas financieras en proyectos de infraestructura.

- Opciones reales: evaluación de proyectos de infraestructura con opciones reales.

- Tasa de descuento: construcción de la tasa de descuento para evaluar proyectos de infraestructura.

- Renegociación de contratos: evidencias empíricas de los motivos que inducen a las renegociaciones.

- Gobierno corporativo: relación del gobierno corporativo con los diferentes esquemas de APPs.

- Financiación: evaluar los bonos de proyectos (project bonds) como instrumento financiero para fortalecer el mercado de capitales y financiar proyectos de infraestructura.

- Fondos de pensiones: creación de mecanismo que incentiven la financiación de proyectos de infraestructura, con foco especial en Colombia.

- Fondos de capital privado: medir el impacto en la financiación de proyectos de infraestructura. 
- Banca multilateral: efecto del financiamiento por medio de la banca multilateral y su impacto en la gestión del riesgo y costo financiero.

- Indicadores de servicio: caracterizar los principales mecanismos utilizados para medir la bondad de los servicios prestados en los proyectos de infraestructura.

- Esquema APPs: metodologías para seleccionar el tipo de esquema óptimo de acuerdo al tipo de proyecto.

- Calificación de riesgo: explorar y caracterizar las metodologías utilizadas por las firmas calificadoras para el establecimiento de la calificación de proyectos de infraestructura.

\section{Referencias}

ANI Agencia Nacional de Infraestructura de Colombia (2012). Matriz de riesgos. http://www.ani.gov.co/sites/default/files/ matriz_de_riesgos_0.pdf

Albújar, A. (2010). El Project Finance: Una técnica para viabilizar proyectos de infraestructura. Documento de trabajo No. 27. Universidad ESAN, Lima.

Akintoye, A. and Beck, M. (2009). Policy, Finance \& Management for Public-Private Partnerships. Wiley-Blackwell

Akintoye, A. and Chinyio, E. (2005). Private Finance Initiative in the healthcare sector: trends and risk assessment. Engineering, Construction and Architectural Management 12(6), 601-616

Alonso-Conde, A., Brown, C. and Rojo-Suarez, J. (2007). Public private partnerships: Incentives, risk transfer and real options. Review of Financial Economics 16(4), 335-349

Aoust, J.M., Bennett, T.C. et Fiszelson, R. (2000). L'analyse et le partage des risques, clé de la réussite d'un partenarial publicprivé. Financement des infrastructures des services collectifs. Le recours au partenarial public-privé, Presses des Fonts et Chaussées, Paris

Aragonés, J., Blanco, C. and Iniesta, F. (2009). Modelización del riesgo de crédito en proyectos de infraestructura. Innovar 19(35), 65-80

Arias, L. (2012). Perspectiva legal de la financiación de proyectos "Project Finance" y el manejo del riesgo. Revista Derecho Privado 23, 211-243
Banco Mundial. (2014). ¿Qué son las asociaciones públicoprivadas? . http://ppp.worldbank.org/public-private-partnership/ es/asociaciones-publico-privadas/definicion

Borgonovoa, E., Gatti, S. and Peccati, L. (2010). What drives value creation in investment projects? An application of sensitivity analysis to project finance transactions. European Journal of Operational Research 205, 227-236

Bovis, C. (2010). Public-private partnerships in the 21st century. ERA Forum 11(3), 379-398

Bracey, N. and Moldovan, S. (2006). Public-Private Partnerships: Risk to the public and private sector. In 6th Global Conference on Business and Economics. Harvard University Symposium

Bravo, S. (2011). Best practice in PPPs Financing. In Best practice in PPPs Financing. Financing Public-Private Partnerships - Best Practices in Latin America. World Bank Institute and Public-Private Infrastructure Advisore Facility Symposium. Washington DC

Comisión de las Comunidades Europeas (2004). Libro verde sobre la colaboración público-privada y el derecho comunitario en materia de contratación pública y concesiones.

Congreso de Colombia (2012a). Ley 1508 de 2012: Por la cual se establece el regimen jurídico de las Asociaciones PúblicoPrivadas. Diario Oficial.

Congreso de Colombia (2012b). Decreto 1467 de 2012. Por la cual se reglamenta la Ley 1508 de 2012. Diario Oficial.

Cruz, C., Marques, R. and Cardoso, P. (2014). Empirical Evidence for Renegotiation of PPP Contracts in the Road Sector. Journal of Legal Affairs and Dispute Resolution in Engineering and Construction 6, 05014003-9

Demirag, I., Khadaroo, I., Stapleton, P. and Stevenson, C. (2011). Risks and the financing of PPP: Perspectives from the financiers. The British Accounting Review, 43(4), 294-310

Dixon, T., Pottinger, G. and Jordan, A. (2005). Lessons from the private finance initiative in the UK: benefits, problems and critical success factors. Journal of Property Investment \& Finance 23(5), 412-423

DNP Departamento Nacional de Planeación de Colombia (2014). Informe trimestral del Registro Único de Asociaciones PúblicoPrivadas (RUAPP). Boletín Núm. 2. 
DNP Departamento Nacional de Planeación de Colombia (2011a). Guía de buenas prácticas para la ejecución de proyectos de Asociación Público-Privada.

DNP Departamento Nacional de Planeación de Colombia. (2011b). Plan Nacional de desarrollo 2010-2014. Prosperidad para todos.

Durán, R. (2006). Project finance y emisión de títulos: dos alternativas de financiación. Editorial y Comunicaciones Universidad Santo Tomas

Engel, E., Fischer, R. and Galetovic, A. (2010). The economics of infrastructure finance: Public-Private Partnerships versus public provision. EIB PAPERS, 40-68

Engel, E., Fischer, R. and Galetovic, A. (2013). The basic Public Finance of Public-Private Partnerships. Journal of the European Economic Association 11(1), 83-111

Espinoza, D. and Morris, J. (2013). Decoupled NPV: a simple, improved method to value infrastructure investments. Construction Management and Economics 31(5), 471-496

Esquivel, F. (2013). Lineamientos para diseñar un estado de la cuestión en investigación educativa. Revista Educación 37(1), $65-87$

Esty, B. (2000). Big deals: financing large-scale investments. Working Knowledge: A Report on Research at Harvard Business School 4(2)

Esty, B. (2003). The Economic Motivations for Using Project Finance. Working Paper. Boston: Harvard University.

Esty, B. (2004). Why study large projects? An introduction to research on project finance. European Financial Management 10(2), 213-224

Esty, B. and Christov, I. (2002). An overview of Project Finance -2002 Update. Working Paper. Boston: Harvard University.

Farquharson, E., Torres de Mästle, C., Yescombe, E. R. and Encinas, J. (2011). How to Engage with the Private Sector in Public-Private Partnerships in Emerging Markets. World Bank

Fay, M. and Marrison M. (2006). Infrastructure in Latin America and the Caribbean. Recent Developments and Key Challenges, Washington, D.C., World Bank. CEPAL

Federal Highway Administration (2007). User Guidebook on Implementing Public-Private Partnerships for Transportation Infrastructure Projects in The United States.
Fedesarrollo (2012). Tendencia económica. Actualidad: Infraestructura de transporte en Colombia: ¿Luz al final del túnel? Gatti, S. (2013). Project Finance in Theory and Practice: Designing, Structuring, and Financing Private and Public Projects. Elsevier. 2nd

Ghersi, H. and Sabal, J. (2006). An introduction to project finance in emerging markets. Estudio IESA No. 29. Working Paper. Caracas: IESA.

Girardone, C. and Snaith, S. (2011). Project finance loan spreads and disaggregated political risk. Applied Financial Economics 21(23), 1725-1734

Gómez, D. and Jurado, J. (2001). Financiación global de proyectos: Project finance. ESIC Editorial

González, J. (2006). La financiación de la colaboración públicoprivada: El «Project Finance». Presupuesto y Gasto Público 45(4), 175-185

Grajales, D. (2012). Estimaciones de valor en grandes proyecto de infraestructura. IV Simposio Internacional de Economía y Finanzas, Universidad EAFIT, Medellín, Colombia

Grimsey, D. and Lewis, M. (2002). Evaluating the risks of public private partnerships for infrastructure projects. International Journal of Project Management 20(2), 107-118

Grimsey, D. and Lewis, M. (2005). Are public private partnerships value for money?. Accounting Forum 29(4), 345-378

Guasch, J.L. (2014). APPs: Experiencia internacional. Mejores prácticas, oportunidades y desafios. Curso Análisis costo beneficio para Asociaciones Público-Privadas.

Guasch, J.L., Benitez, D., Portabales, I. and Flor, L. (2014). The renegotiation of PPP Contracts: An overview of its recent evolution in Latin America. International Transport Forum.

Guasch, J. L. (2004). Concesiones en infraestructura: cómo hacerlo bien. práctico. World Bank, $5^{\text {ta }}$ edición

Guasch, J. L. and Spiller, P. (2001). Managing the regulatory process: design, concepts, issues and the latin American Caribbean Story. World Bank.

Gutiérrez, M. y Bielenberg, G. (2009). ProjectFinance. Principios y Aplicaciones. En Latin Finance, Cumbre de la Infraestructura y Finanzas Sub-soberanas. Banco Interamericano de Desarrollo, Ixtapan de la Sal, México 
Hinojosa, S. (2009). Opciones reales en inversiones públicas: Un eslabón que faltaba. Revisión de literatura y enfoque conceptual. Documento de trabajo No. 21. Working Paper. Lima: Universidad ESAN.

Hinojosa, A. (2010). Un indicador de elegibilidad para seleccionar proyectos de Asociaciones Público-Privadas en infraestructura y servicios.

Hodge, G. and Greve, C. (2005). Introduction. En G. Hodge and C. Greve (Eds.), The Challenge of Public-Private Partnerships: Learning From International Experience. Edward Elgar

Hoon, Y., Chih, Y. and Ibbs, C. (2009). Towards a comprehensive understanding of public private partnerships for infrastructure development. California Management Review 51(2), 51-78

Iyer, K. and Sagheer, M. (2012). Optimization of Bid-Winning Potential and Capital Structure for Build-Operate-Transfer Road Projects in India. Journal of Management in Engineering 28(2), 104-113

Jensen, M. (1986). Agency Cost of Free Cash Flow, Corporate Finance and Takeovers. American Economic Review 76(2), 323-329

Jensen, M. C. and Meckling, W. (1976). Theory of the Firm: Managerial Behavior, Agency Costs and Ownership Structure. Journal of Financial Economics 3, 305-360

Jin, X.-H. and Zhang, G. (2011). Modelling optimal risk allocation in PP projects using artificial neural networks. International Journal of Project Management 29(5), 591-603

Kayser, D. (2013). Recent Research in Project Finance-A Commented Bibliography. Procedia Computer Science 17, 729-736

Kleimeier, S. (1995). Limited and Norecourse. Project Finance: a Survey. Estudios de Administración 2(1), 27-67

Kleimeier, S. and Versteeg, R. (2010). Project finance as a driver of economic growth in low-income countries. Review of Financial Economics 19(2), 49-59

Liu, J., Love, P., Davis, P., Smith, J. and Regan, M. (2014). Conceptual Framework for the Performance Measurement of Public-Private Partnerships. Journal of Infrastructure Systems, 04014023

López, J. and García, P. (2005). Finanzas en el mundo corporativo. Un enfoque práctico. McGraw Hill
Martins, J., Marques, R. and Cruz, C. (2013). Real Options in Infrastructure: Revisiting the Literature. Journal of Infrastructure Systems, 04014026

Mascareñas, J. (2011). Fusiones, adquisiciones y valoración de empresas. ECOBOOK

Mascareñas, J. (1999). Innovación Financiera: Aplicaciones para la gestión empresarial. McGraw-Hill

Molina, H. y Del Carpio, J. (2004). Financiamiento de Inversiones mediante el Project Finance. Revista de la Facultad de Ingeniería Industrial 7(2), 76-82

Morin, E. (2014). Regulación de niveles de servicio. Las Asociaciones Público-Privadas para el desarrrollo de infraestructura y servicios. PIAPPEM, México

Moszoro, M. and Gasiorowski, P. (2008). Optimal Capital Structure of Public-Private Partnerships (No. 08/1). IMF Working Papers. International Monetary Fund

Mouraviev, N. and Kakabadse, N. (2012). Conceptualising public-private partnerships: A critical appraisal of approaches to meanings and forms. Society and Business Review 7(3), 260-276

Perroti, D. and Sánchez, R. (2011). La brecha de infraestructura en América Latina y el Caribe. CEPAL - Serie Recursos naturales e infraestructura $\mathrm{N}^{\circ} 153$.

Pöll, E. (2012). Public Real State. Understanding German Real Estate Markets. Edited by T. Just and W. Maennig. Berlin: Springer

Rebeiz, K. (2012). Public-Private Partnership Risk Factors in Emerging Countries: BOOT Illustrative Case Study. Journal of Management in Engineering 28(4), 421-428

Rozas, P., Bonifaz, J. and Guerra-García, G. (2012). El financiamiento de la infraestructura. Propuestas para el desarrollo sostenible de una politica sectorial. Naciones Unidas. CEPAL.

Rosillo, J. y Perdono, J. (2012). Modelo de programación matemática para determinar la estructura financiera óptima en inversiones financiadas mediante Project Finance. XII International Finance Conference, Universidad EAFIT, Medellín, Colombia

Saussier, S. (2013). Public-private partnerships. Journal of Economic Behavior \& Organization 89, 143-144 
Suárez, J. (1993). Project Finance: Financiación de un Parque Eólico. Caso de estudio. IESE Business School Universidad de Navarra.

Svědík, J. and Tetřevová, L. (2012). Financing and Mezzanine Capital in the Context of PPP Projects in the Czech Republic. Recent Research in Business and Economics, WSEAS Press, 113-117

Tang, L., Shen, Q. and Cheng, E. (2010). A review of studies on Public-Private Partnership projects in the construction industry. International Journal of Project Management 28(7), 683-694

The Economist Intelligent Unit (2013). Evaluando el entorno para las Asociaciones Público-Privadas en América Latina y el Caribe. Infrascope 2012

Thomson, C., Goodwin, J. and Yescombe, E. R. (2005). Evaluation of PPP projects financed by the EIB. European Invesment Bank.

Vasallo, A.M. (2012). El sector de construcción y obras públicas: la necesidad de nuevos modelos de financiación. Revista de Obras Públicas 159(3537), 77-92

Vasallo, J.M. and Izquierdo, R. (2010). Infraestructura pública y participación privada. Conceptos y experiencias en América y España. Corporación Andina de Fomento CAF

Vega, L. (2008). El "Project Finance" (I/II) Conceptos básicos. Revista de La Asociación de Ingenieros I.C.A.I., LXXXV(IV), 61-65
Vives, A. Paris, A. y Benavides, J. (2007). Estructuración financiera de proyectos de infraestructura en asociaciones público-privadas: Una aplicación a proyectos de agua $\mathrm{y}$ saneamiento. Banco Interamericano de Desarrollo.

World Bank (2014). Sources of Financing. http://ppp.worldbank. org/public-private-partnership/financing/sources\#mezzanine

World Bank (2012). Green Infrastructure Finance: Framework Report. World Bank. https://openknowledge.worldbank.org/ handle/10986/9367

Xiong, W. and Zhang, X. (2014). Concession Renegotiation Models for Projects Developed through Public-Private Partnerships. Journal of Construction Engineering and Management 140(5), 04014008

Yescombe, E. R. (2007). Public-Private Partnerships: Principles of Policy and Finance. Elsevier

Zhang, X. and Chen, S. (2013). A systematic framework for infrastructure development through public private partnerships. IATSS Research 36(2), 88-97

Zhang, Z. and Durango-Cohen, P. (2012). A strategic model of public-private partnerships in transportation: Effect of taxes and cost structure on investment viability. Research in Transportation Economics 36(1), 9-18 